\title{
Selective Direct Synthesis of Trialkoxysilanes in a Packed Bed Flow Tubular Reactor
}

Fidelis Chigondo, Paul Watts ${ }^{*}$ and Ben Zeelie

InnoVenton: Institute for Chemical Technology, Nelson Mandela Metropolitan University, P O Box 77000, Port Elizabeth, 6031, South Africa.

E-mail: Paul.Watts@nmmu.ac.za. Tel: +27 41504 3694.Fax: +27 415049281

Number of pages: 20

Number of figures: 30

Number of tables: 3

\section{EXPERIMENTAL}

\section{Gas Chromatography (GC) method}

Analysis of samples by GC was performed using an Agilent GC (6850) with a DB-5 $(30 \mathrm{~cm})$ column, (Agilent J\&W, USA) and high purity nitrogen (99.997\%, Afrox, South Africa) as carrier gas. Samples were analysed using the following method; injector temperature $250^{\circ} \mathrm{C}$, Flame Ionization Detector (FID) temperature $300{ }^{\circ} \mathrm{C}$, carrier gas flow rate $1 \mathrm{ml} / \mathrm{min}$, oven temperature $70{ }^{\circ} \mathrm{C}$ for 0 min then ramped firstly to $90{ }^{\circ} \mathrm{C}$ at $3{ }^{\circ} \mathrm{C} \mathrm{min} / \mathrm{min}$ and secondly to $220^{\circ} \mathrm{C}$ at $20^{\circ} \mathrm{C} / \mathrm{min}$ and held for $3 \mathrm{~min}$.

\section{Gas Chromatography-Mass Spectrometry (GC-MS) method}

Analysis of samples by GC was performed using a Thermo Finnigan spectrometer with an EI ionisation source, a DB-5 $(30 \mathrm{~cm})$ column, (Agilent J\&W, USA) and high purity helium (99.997\%, Afrox, South Africa) as carrier gas. Samples were analysed using the following method; injector temperature $250^{\circ} \mathrm{C}$, carrier gas flow rate $1 \mathrm{ml} / \mathrm{min}$, oven temperature $70{ }^{\circ} \mathrm{C}$ for 0 min then ramped firstly to $90{ }^{\circ} \mathrm{C}$ at $3{ }^{\circ} \mathrm{C} \mathrm{min} / \mathrm{min}$ and secondly to $220{ }^{\circ} \mathrm{C}$ at $20{ }^{\circ} \mathrm{C} / \mathrm{min}$ and held for $3 \mathrm{~min}$. 


\section{Experimental setup}

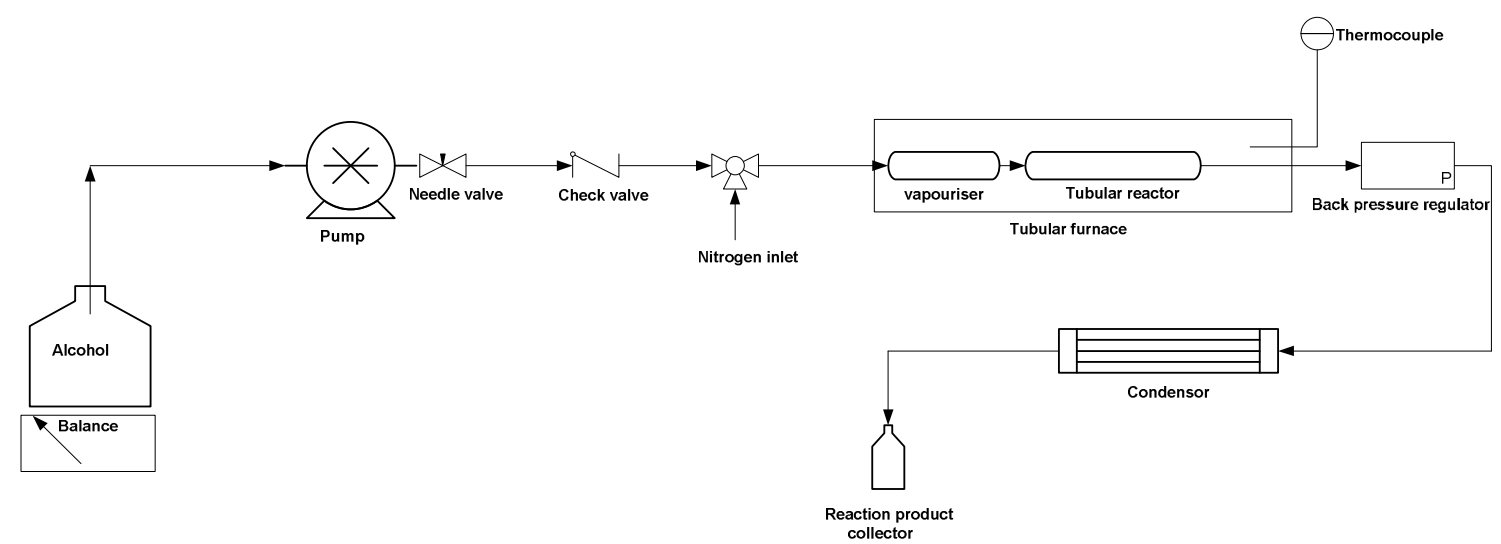

Figure S1. Packed bed flow tubular reactor rig schematic flow diagram.

\section{CHARACTERIZATION OF PRODUCTS}

\section{Trimethoxysilane}

$v_{\max } / \mathrm{cm}^{-1} 2947,2844,2199,1460,1192,1073,864,786,700$ and 449

$\delta_{\mathrm{H}}\left(400 \mathrm{MHz}, \mathrm{CDCl}_{3}\right) 3.61(9 \mathrm{H}, \mathrm{s})$ and $4.23(1 \mathrm{H}, \mathrm{s})$

$\delta_{\mathrm{C}}\left(100 \mathrm{MHz}, \mathrm{CDCl}_{3}\right) 50(3 \mathrm{C})$

$m / z(\mathrm{EI}) 123\left(\mathrm{M}^{+}, 7 \%\right), 122(8), 121(95), 91(100), 61$ (72) and 59 (72)

$\mathrm{GC} t_{\mathrm{R}}=2.29 \mathrm{~min}$

$\mathrm{GC}-\mathrm{MS} t_{\mathrm{R}}=1.01 \mathrm{~min}$

\section{Tetramethoxysilane}

$v_{\max } / \mathrm{cm}^{-1} 2947,2844,1461,1192,1072,813$ and 641

$\delta_{\mathrm{H}}\left(400 \mathrm{MHz}, \mathrm{CDCl}_{3}\right) 3.32(12 \mathrm{H}, \mathrm{s})$

$\delta_{\mathrm{C}}\left(100 \mathrm{MHz}, \mathrm{CDCl}_{3}\right) 50.4(4 \mathrm{C})$

$m / z(E I) 154\left(\mathrm{M}^{+}+1,8 \%\right), 153\left(\mathrm{M}^{+}, 38 \%\right), 152$ (100), $151(34), 121$ (28), 91 (9) and 90 (5)

$\mathrm{GC} t_{\mathrm{R}}=3.09 \mathrm{~min}$

GC-MS $t_{\mathrm{R}}=1.35 \mathrm{~min}$ 


\section{Triethoxysilane}

$v_{\max } / \mathrm{cm}^{-1} 2976,2928,2886,2184,1484,1443,1391,1295,1167,1100,1072,958,853,808$, 767,696 and 475

$\delta_{\mathrm{H}}\left(400 \mathrm{MHz}, \mathrm{CDCl}_{3}\right) 1.20(9 \mathrm{H}, \mathrm{t}, J=7.2), 3.80(6 \mathrm{H}, \mathrm{q}, J=7.0)$ and $4.23(1 \mathrm{H}, \mathrm{s})$

$\delta_{\mathrm{C}}\left(100 \mathrm{MHz}, \mathrm{CDCl}_{3}\right) 18.0(3 \mathrm{C})$ and $59.0(3 \mathrm{C})$

$m / z(\mathrm{EI}) 165\left(\mathrm{M}^{+}, 5 \%\right), 264$ (10), 163 (74), 149 (92), 119 (100), 105 (92) and 91 (55)

$\mathrm{GC} t_{\mathrm{R}}=3.64 \mathrm{~min}$

GC-MS $t_{\mathrm{R}}=2.81 \mathrm{~min}$

\section{Tetraethoxysilane}

$v_{\max } / \mathrm{cm}^{-1} 2975,2929,2890,1484,1442,1391,1366,1296,1168,1100,1072,957,783,654$ and 470

$\delta_{\mathrm{H}}\left(400 \mathrm{MHz}, \mathrm{CDCl}_{3}\right) 1.19(12 \mathrm{H}, \mathrm{t}, J=7.3)$ and $3.79(8 \mathrm{H}, \mathrm{q}, J=7.1)$

$\delta_{\mathrm{C}}\left(100 \mathrm{MHz}, \mathrm{CDCl}_{3}\right) 18.0(4 \mathrm{C})$ and $58.2(4 \mathrm{C})$

$m / z(\mathrm{EI}) 209$ ( $\left.\mathrm{M}^{+}, 10 \%\right), 194$ (12), 193 (100), 163.1 (52), 149 (85) and 119 (37),

$\mathrm{GC} t_{\mathrm{R}}=6.63 \mathrm{~min}$

GC-MS $t_{\mathrm{R}}=5.27 \mathrm{~min}$

\section{Tripropoxysilane}

$v_{\max } / \mathrm{cm}^{-1} 2974,2930,2879,2192,1443,1391,1367,1295,1167,1074,1019,910,853,767$, 747, 696 and 475

$\delta_{\mathrm{H}}\left(400 \mathrm{MHz}, \mathrm{CDCl}_{3}\right) 0.91(9 \mathrm{H}, \mathrm{t}, J=7.4), 1.59(6 \mathrm{H}, \mathrm{sext}, J=7.1), 3.73(6 \mathrm{H}, \mathrm{t}, J=6.7)$ and $4.28(1 \mathrm{H}, \mathrm{s})$

$\delta_{\mathrm{C}}\left(100 \mathrm{MHz}, \mathrm{CDCl}_{3}\right) 10.1(3 \mathrm{C}), 25.5(3 \mathrm{C})$ and $65.0(3 \mathrm{C})$

$m / z$ (EI) 207 ( $\left.\mathrm{M}^{+}, 4 \%\right), 206$ (11), 178 (12), 177 (100), 163 (12), 147 (30), 135 (86), 119 (34),

77 (17) and $63(76)$

$\mathrm{GC} t_{\mathrm{R}}=6.73$

GC-MS $t_{\mathrm{R}}=5.37$

\section{Tetrapropoxysilane}

$v_{\max } / \mathrm{cm}^{-1} 2962,2936,2878,1463,1391,1263,1154,1079,1015,901,844,745$ and 498 $\delta_{\mathrm{H}}\left(400 \mathrm{MHz}, \mathrm{CDCl}_{3}\right) 0.78(12 \mathrm{H}, \mathrm{t}, J=7.4), 1.45(8 \mathrm{H}, \mathrm{sext}, J=7.1)$ and $3.59(8 \mathrm{H}, \mathrm{t}, J=6.4)$ $\delta_{\mathrm{C}}\left(100 \mathrm{MHz}, \mathrm{CDCl}_{3}\right) 9.8(4 \mathrm{C}), 25.3(4 \mathrm{C})$ and $67.8(4 \mathrm{C})$ 
m/z (EI) 265 (M+, 2\%), 264 (5), 237 (6), 236 (16), 235 (100), 193 (30), 151 (37), 121 (22)

and $93(12)$

$\mathrm{GC} t_{\mathrm{R}}=11.55$

GC-MS $t_{\mathrm{R}}=13.15$

\section{Tributoxysilane}

$v_{\max } / \mathrm{cm}^{-1} 2961,2932,2876,2192,1459,1391,1296,1235,1167,1076,1041,1011,959,855$, $802,768,733,696,474$ and 430

$\delta_{\mathrm{H}}\left(400 \mathrm{MHz}, \mathrm{CDCl}_{3}\right) 0.91(9 \mathrm{H}, \mathrm{t}, J=7.3), 1.37(6 \mathrm{H}, \mathrm{sext}, J=7.4), 1.56(6 \mathrm{H}$, quint, $J=7.3)$, $3.77(6 \mathrm{H}, \mathrm{t}, J=6.6)$ and $4.29(1 \mathrm{H}, \mathrm{s})$

$\delta_{\mathrm{C}}\left(100 \mathrm{MHz}, \mathrm{CDCl}_{3}\right) 13.7(3 \mathrm{C}), 18.2(3 \mathrm{C}), 34.5(3 \mathrm{C})$ and $63.2(3 \mathrm{C})$

$m / z$ (EI) 249 ( $\left.\mathrm{M}^{+}, 6 \%\right), 248(8), 247$ (10), 233 (90), 205 (38), 191 (42), 175 (100), 161 (76), $147(32)$ and 135 (37).

$\mathrm{GC} t_{\mathrm{R}}=11.09 \mathrm{~min}$

GC-MS $t_{\mathrm{R}}=6.80 \mathrm{~min}$

\section{Tetrabutoxysilane}

$v_{\max } / \mathrm{cm}^{-1} 2958,2933,2874,1463,1387,1301,1265,1235,1151,1084,1040,1010,989,902$, $836,800,733,522$ and 413

$\delta_{\mathrm{H}}\left(400 \mathrm{MHz}, \mathrm{CDCl}_{3}\right) 0.84(12 \mathrm{H}, \mathrm{t}, J=7.4), 1.30(8 \mathrm{H}, \mathrm{sext}, J=7.2), 1.45-1.49(8 \mathrm{H}$, quint, $J$ $=6.9)$ and $3.68(8 \mathrm{H}, \mathrm{t}, J=6.3)$

$\delta_{\mathrm{C}}\left(100 \mathrm{MHz}, \mathrm{CDCl}_{3}\right) 13.5(4 \mathrm{C}), 18.7(4 \mathrm{C}), 34.3(4 \mathrm{C})$ and $62.9(4 \mathrm{C})$

m/z (EI) 321 ( M+1\%), 320 (2), 219 (2), 279 (6), 278 (18), 277 (100), 263 (12), 221 (24),

205 (12), 165 (22), 123 (23) and 79 (33)

$\mathrm{GC} t_{\mathrm{R}}=14.17 \mathrm{~min}$

GC-MS $t_{\mathrm{R}}=16.44 \mathrm{~min}$ 
Trimethoxysilane ${ }^{1} \mathrm{H}$ NMR, ${ }^{13} \mathrm{C}$ NMR and GC-MS

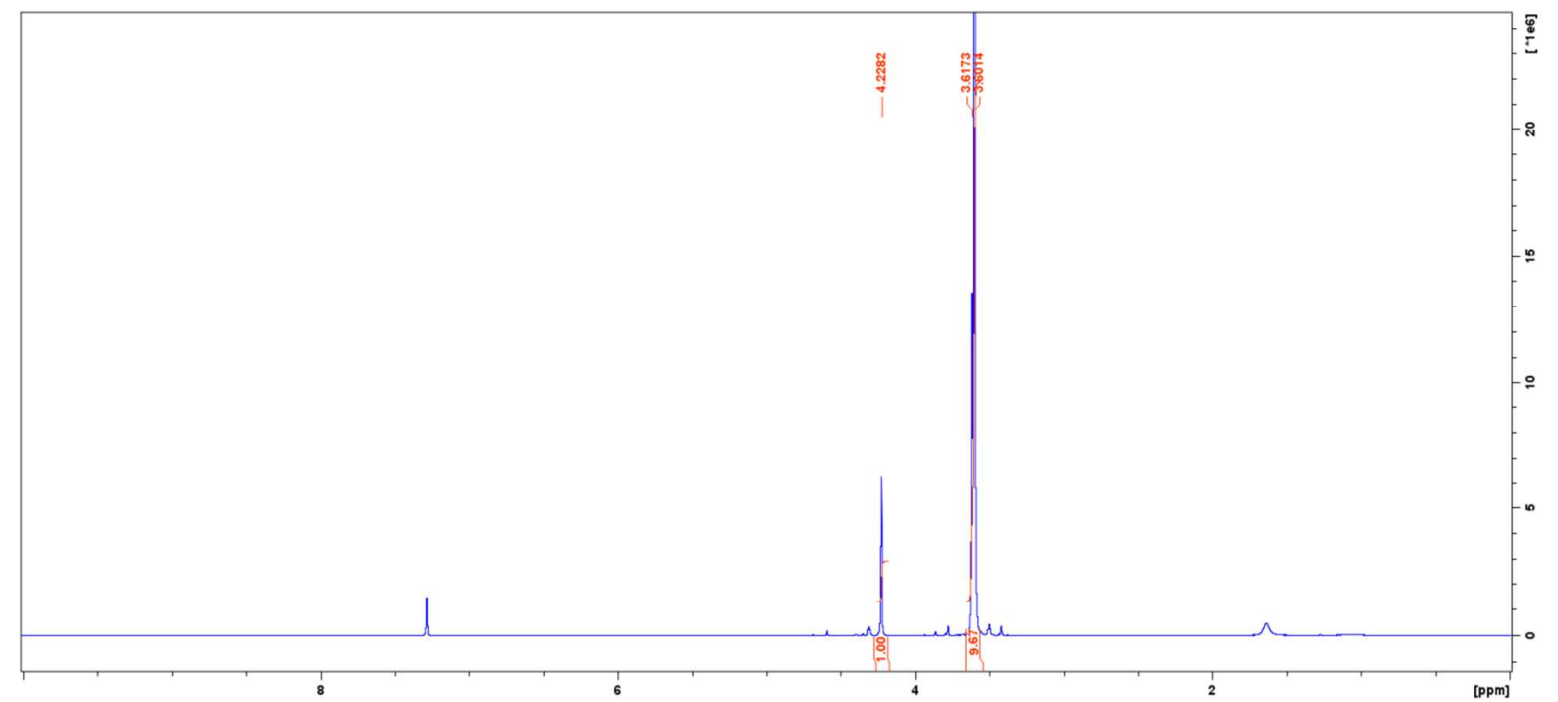

Figure S2. Trimethoxysilane ${ }^{1} \mathrm{H}$ NMR spectrum.

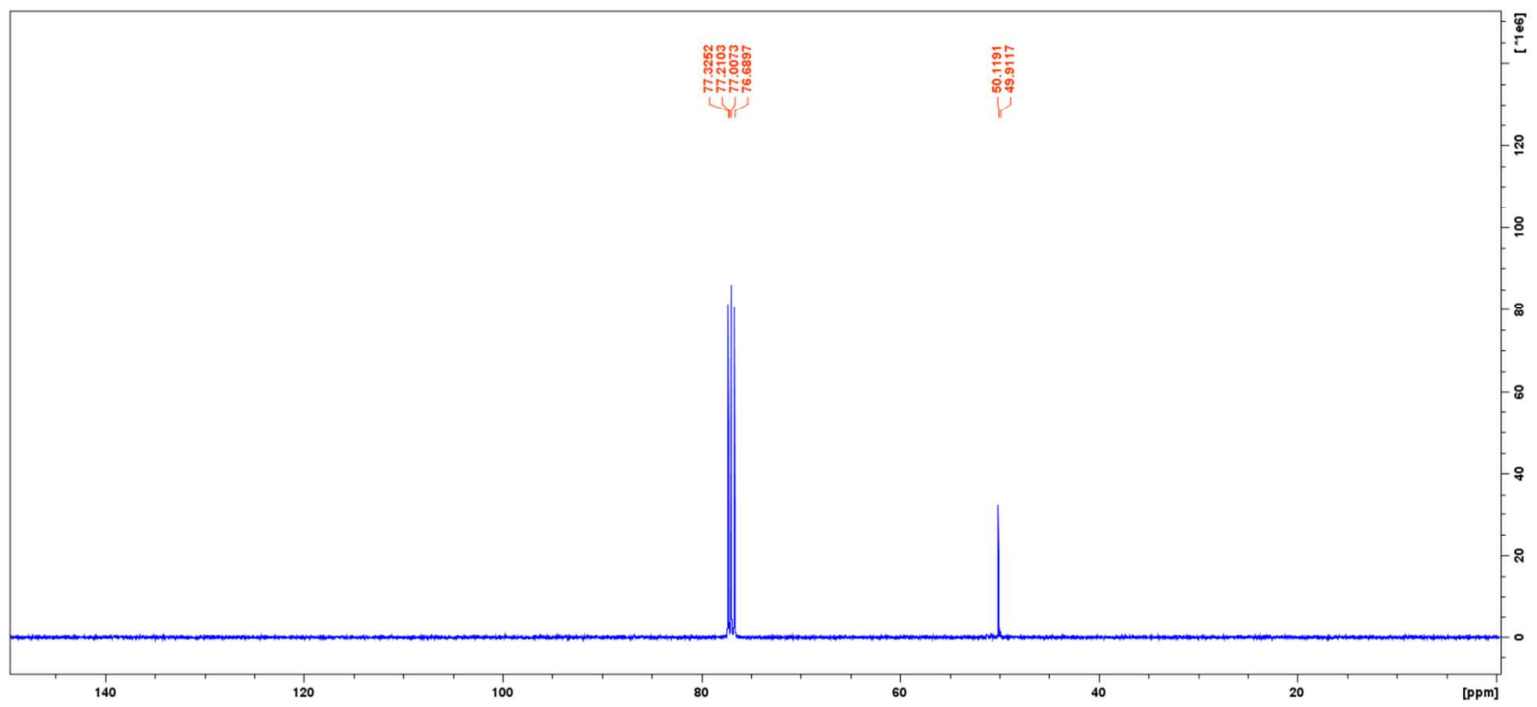

Figure S3. Trimethoxysilane ${ }^{13} \mathrm{C}$ NMR spectrum. 


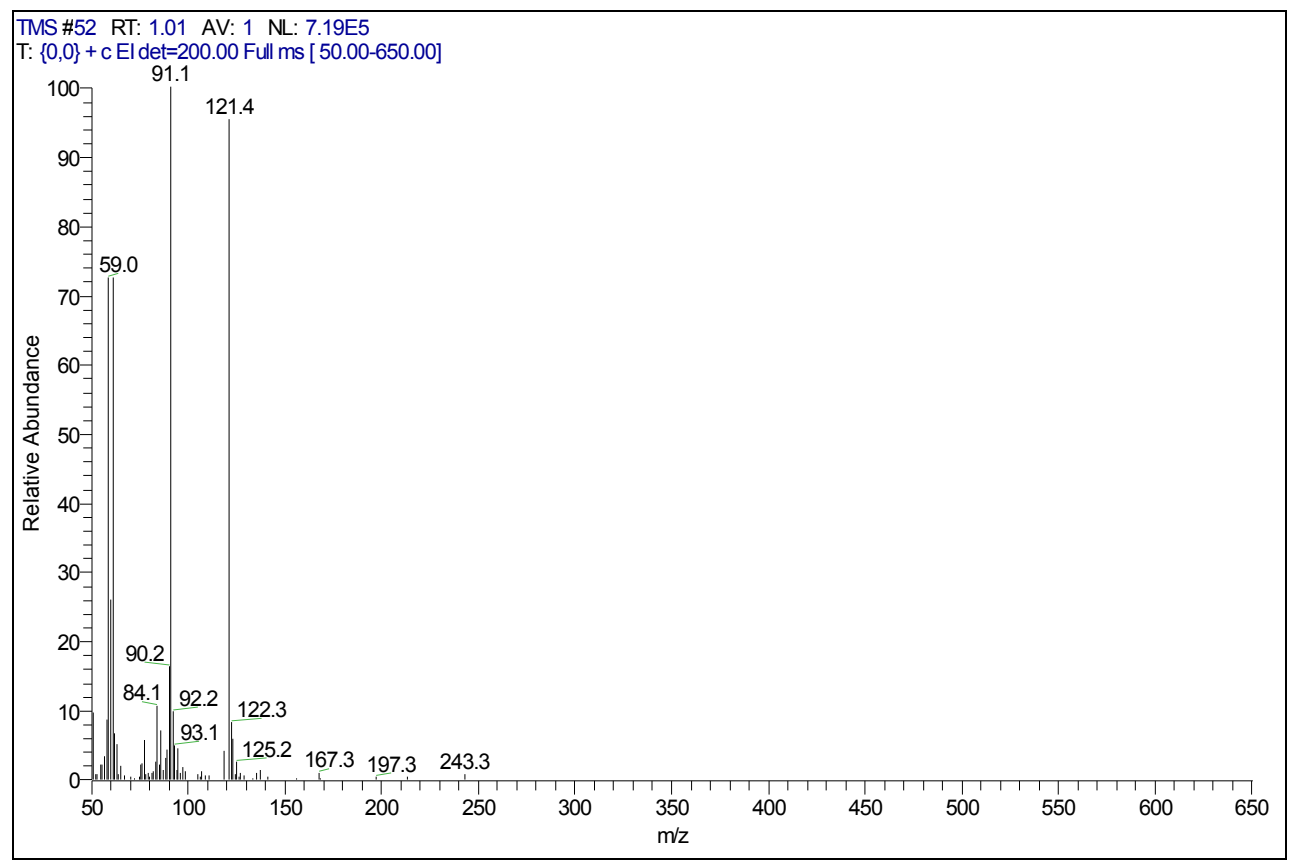

Figure S4. Trimethoxysilane GC-MS spectrum.

Tetramethoxysilane ${ }^{1} \mathrm{H}$ NMR, ${ }^{13} \mathrm{C}$ NMR and GC-MS

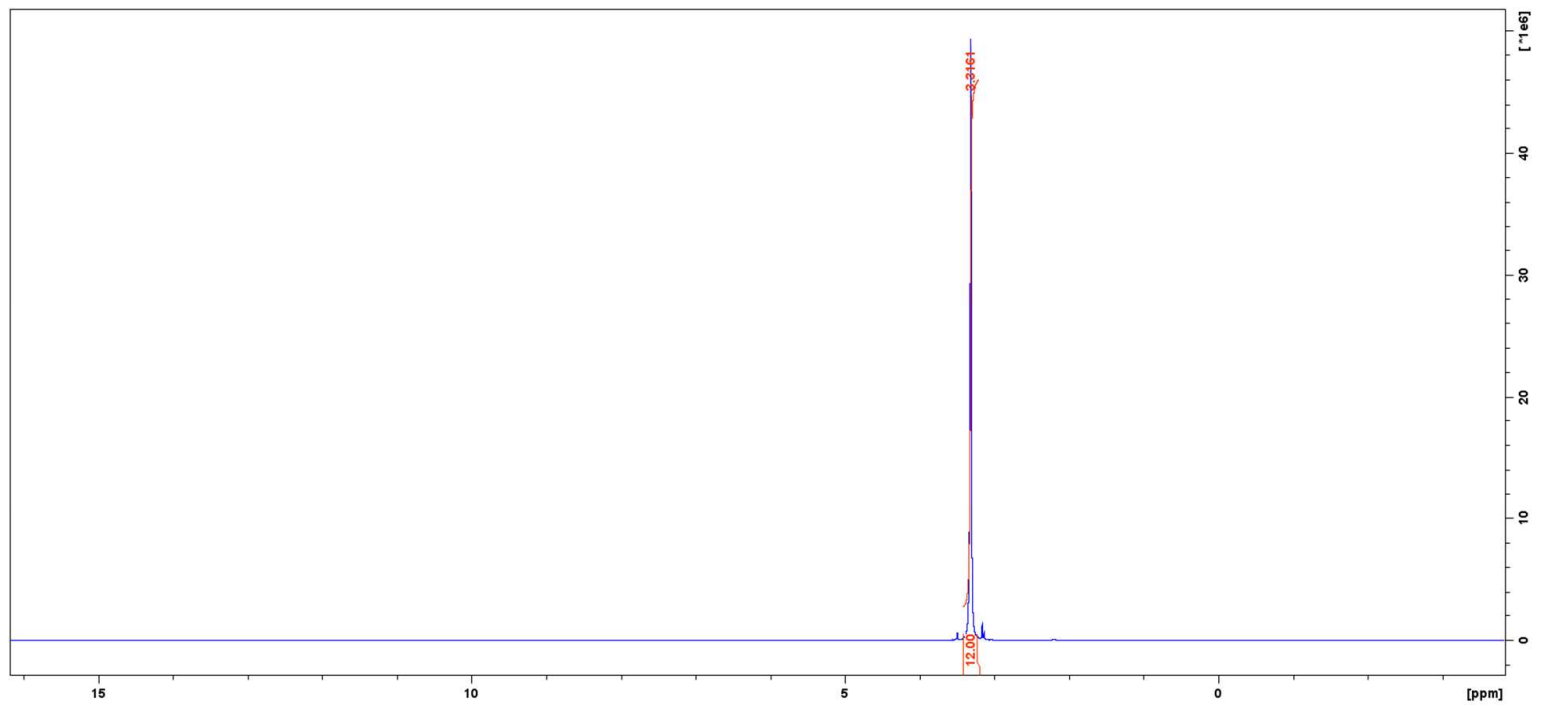

Figure S5. Tetramethoxysilane ${ }^{1} \mathrm{H}$ NMR spectrum. 


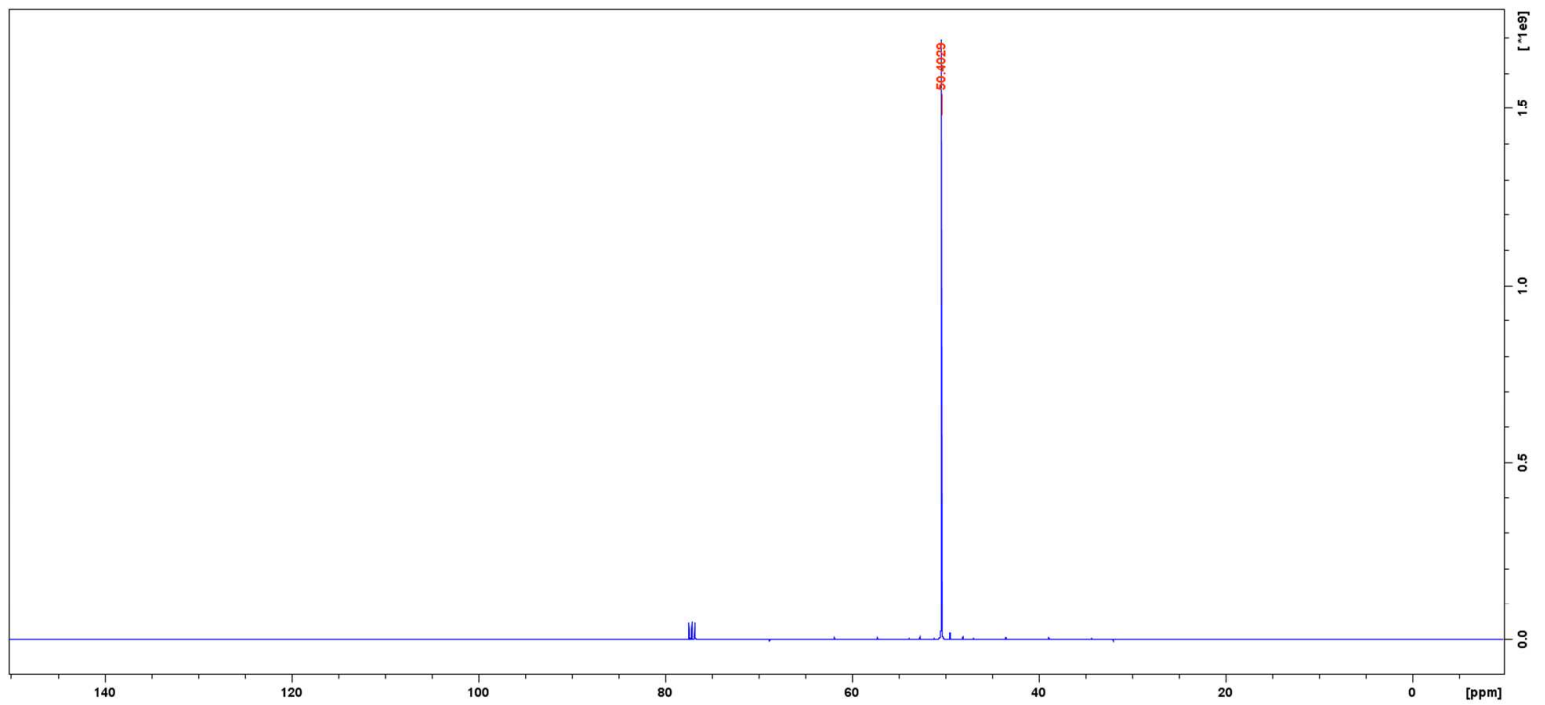

Figure S6. Tetramethoxysilane ${ }^{13} \mathrm{C}$ NMR spectrum.

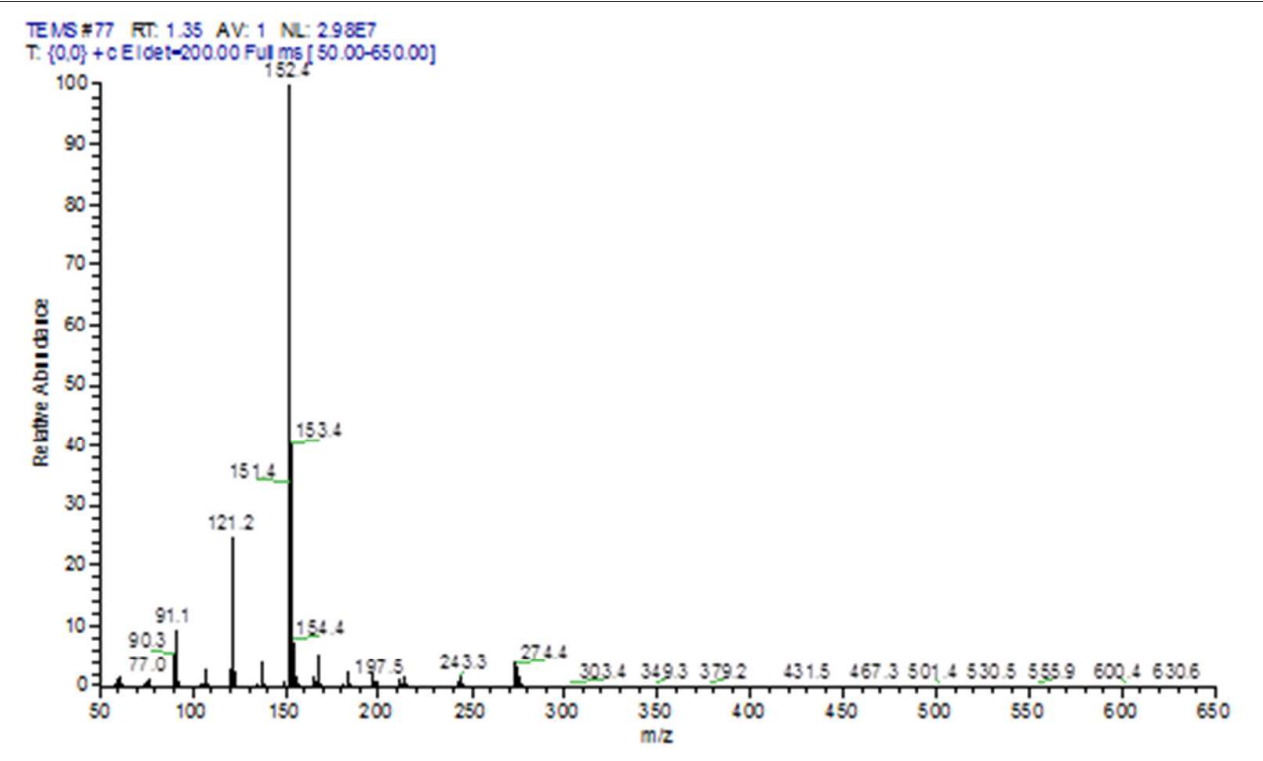

Figure S7. Tetramethoxysilane GC-MS spectrum. 
Triethoxysilane ${ }^{1} \mathrm{H}$ NMR, ${ }^{13} \mathrm{C}$ NMR and GC-MS

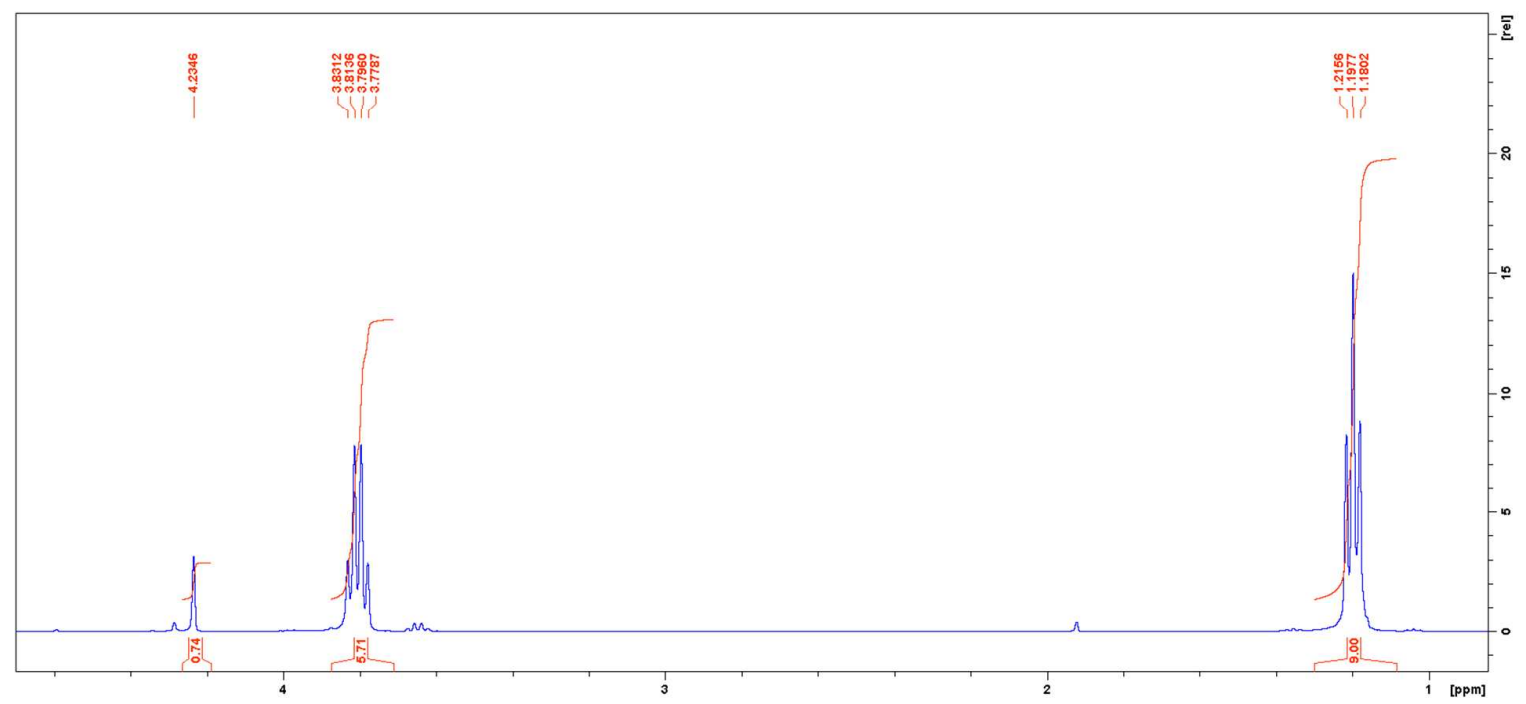

Figure S8. Triethoxysilane ${ }^{1} \mathrm{H}$ NMR spectrum.

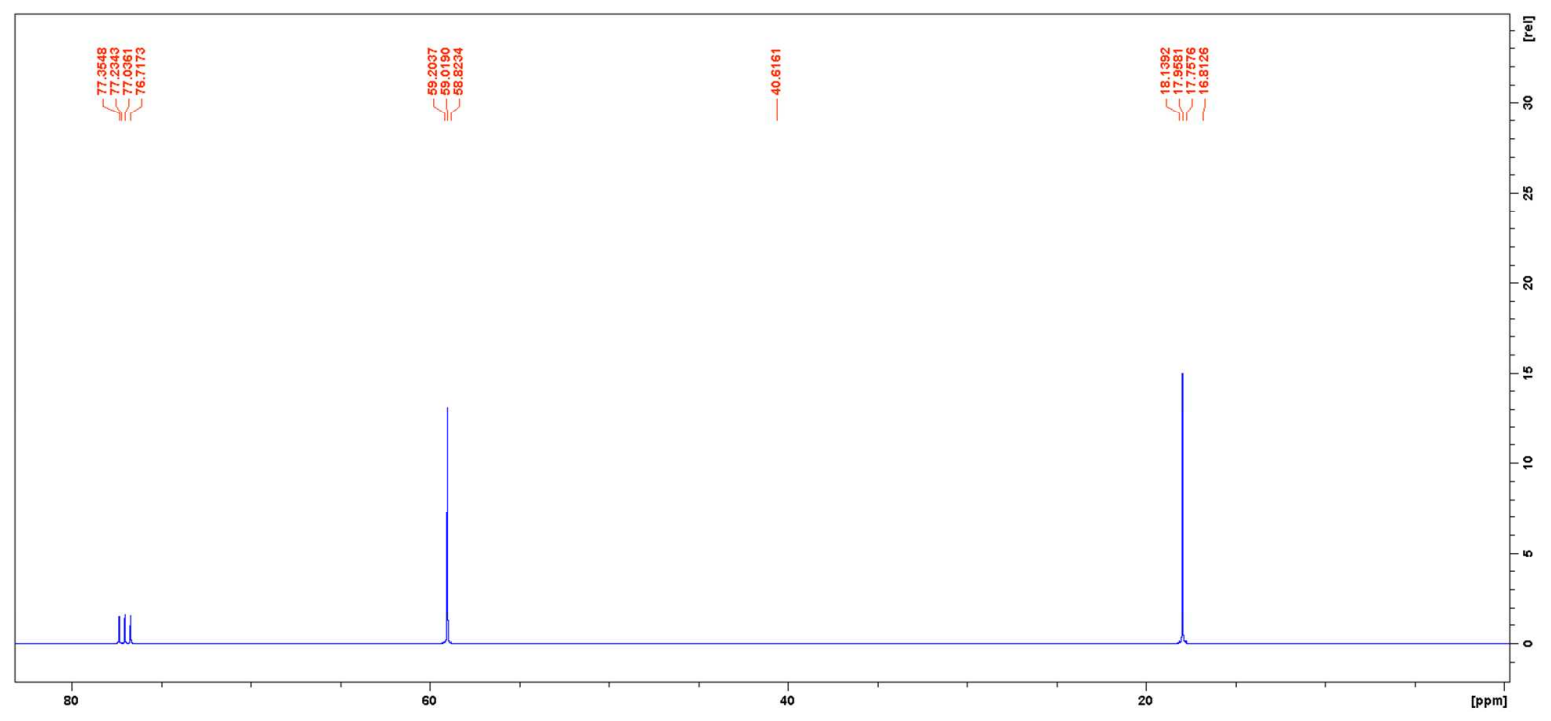

Figure S9. Triethoxysilane ${ }^{13} \mathrm{C}$ NMR spectrum. 


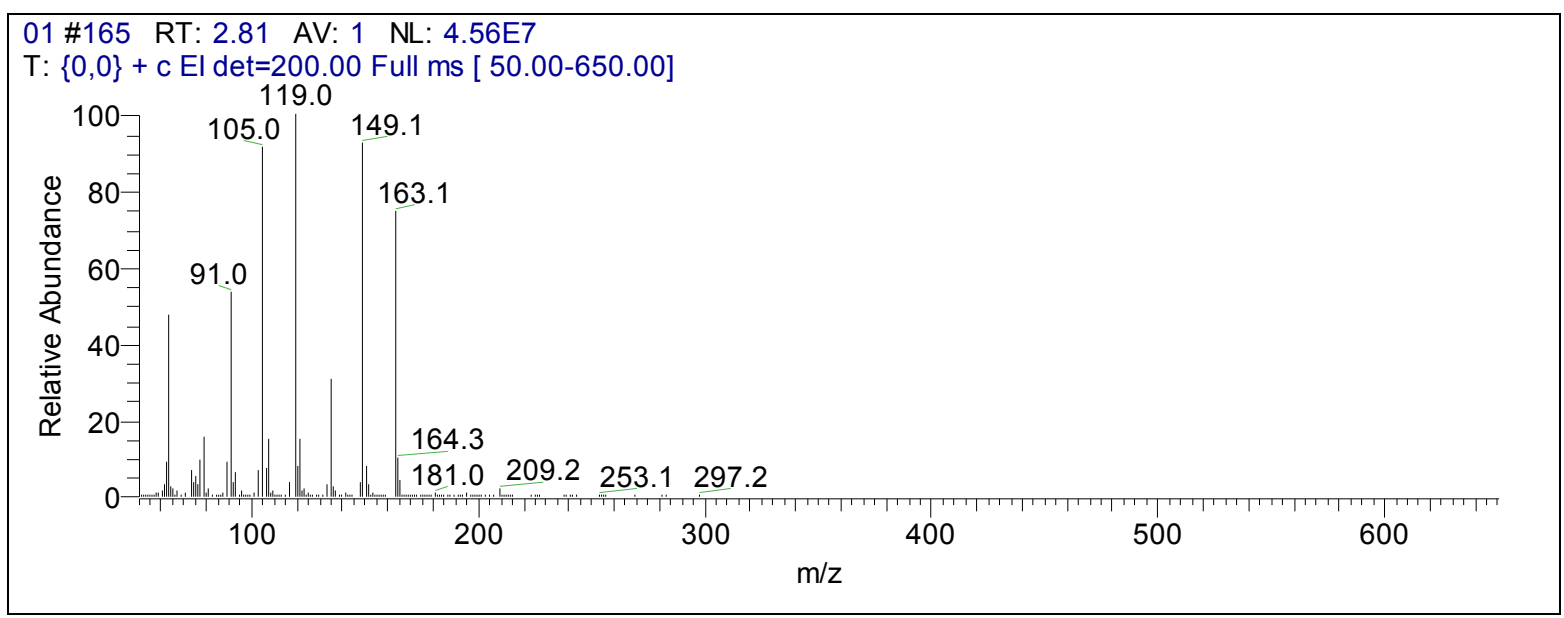

Figure S10. Triethoxysilane GC-MS spectrum.

\section{Tetraethoxysilane ${ }^{1} \mathrm{H}$ NMR, ${ }^{13} \mathrm{C}$ NMR and GC-MS}

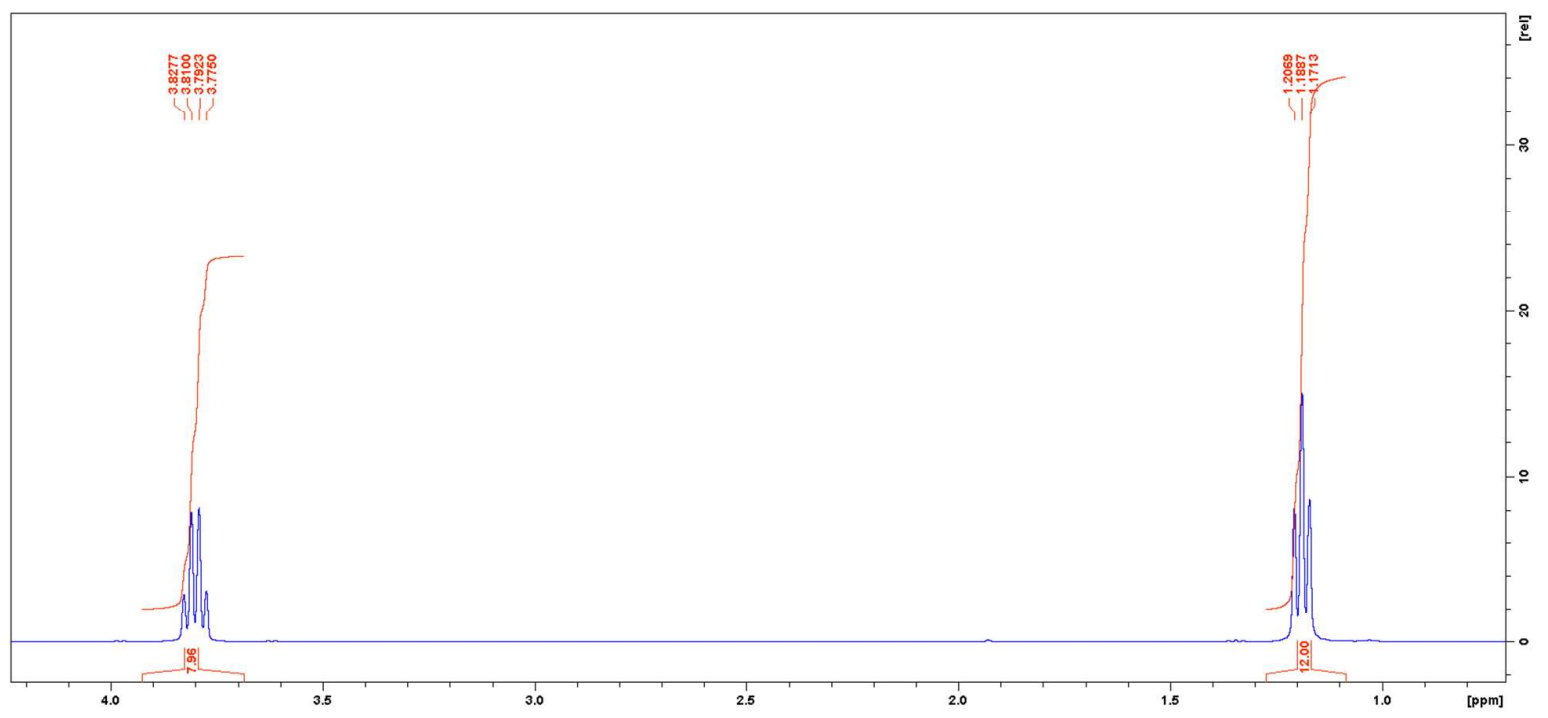

Figure S11. Tetraethoxysilane ${ }^{1}$ H NMR spectrum. 


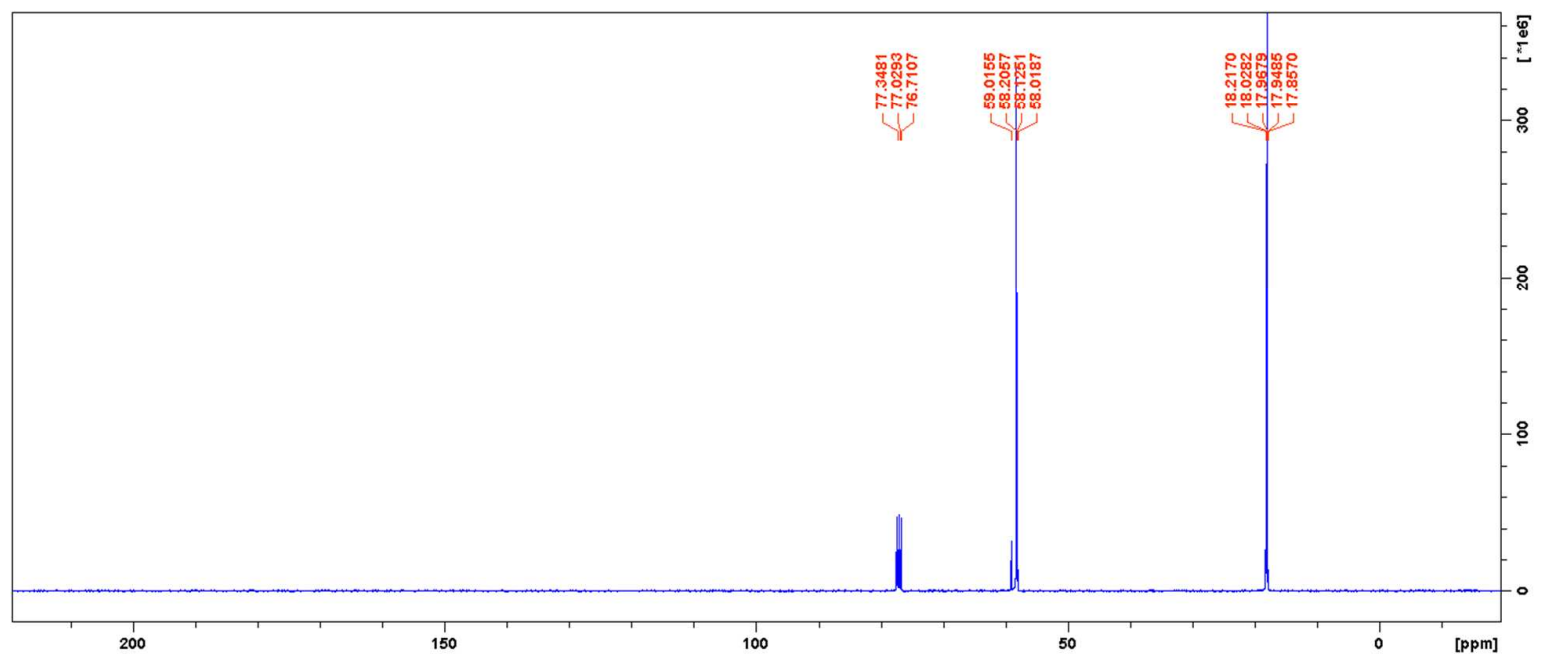

Figure S12. Tetraethoxysilane ${ }^{13} \mathrm{C}$ NMR spectrum.

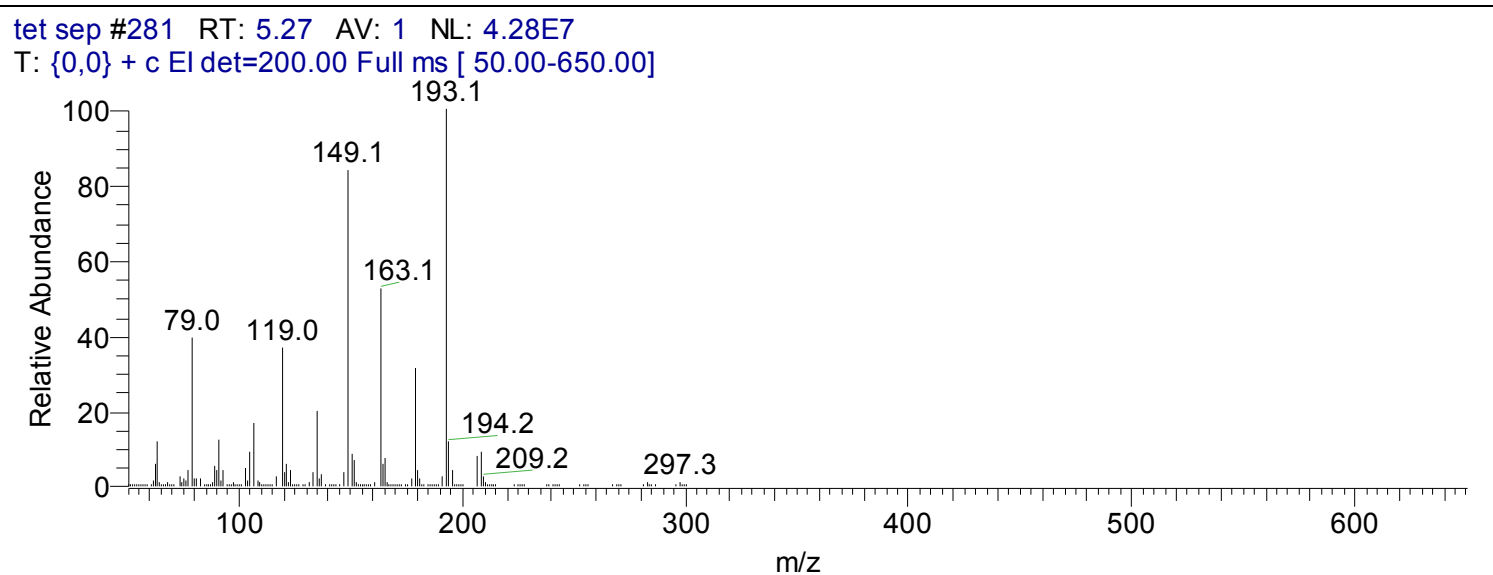

Figure S13. Tetraethoxysilane GC-MS spectrum. 
Tripropoxysilane ${ }^{1} \mathrm{H}$ NMR, ${ }^{13} \mathrm{C}$ NMR and GC-MS

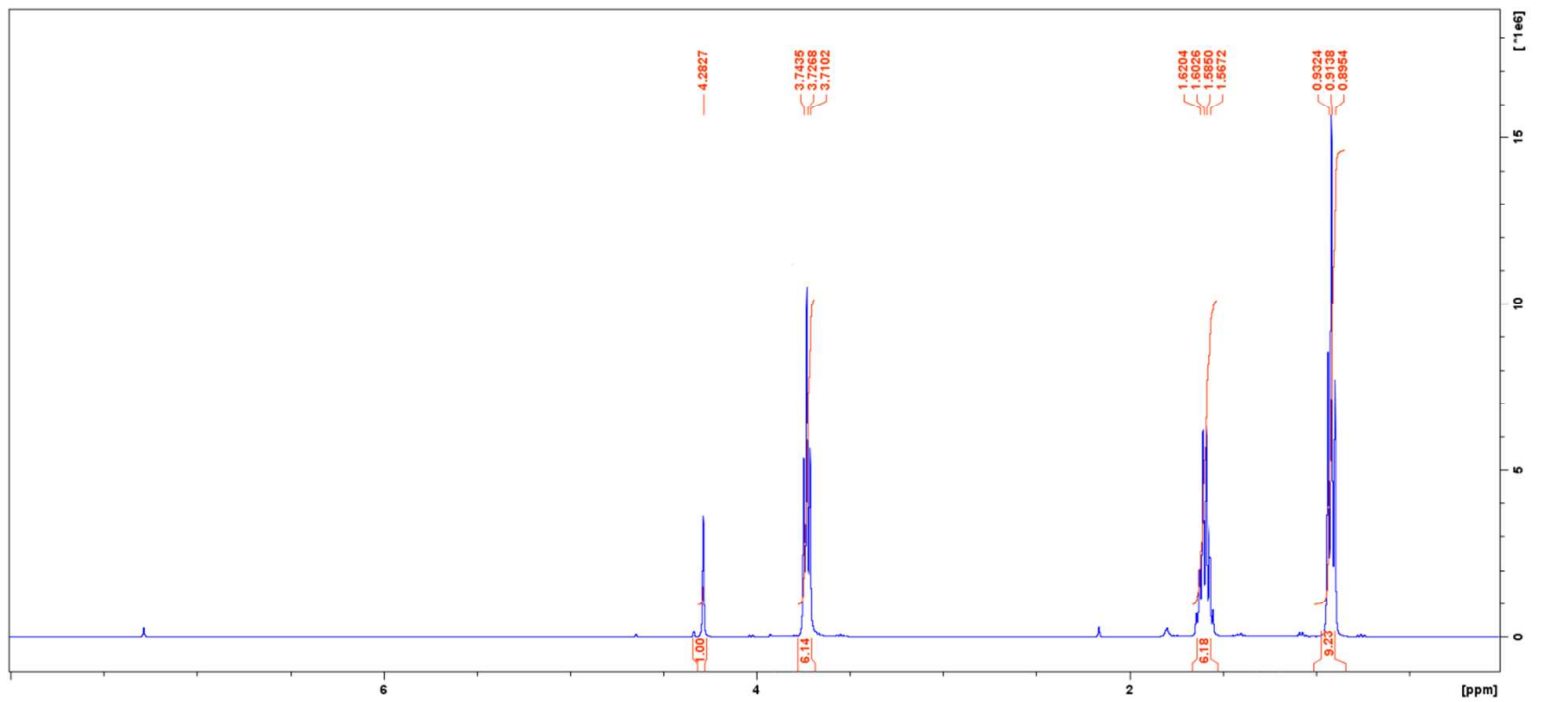

Figure S14. Tripropoxysilane ${ }^{1} \mathrm{H}$ NMR spectrum.

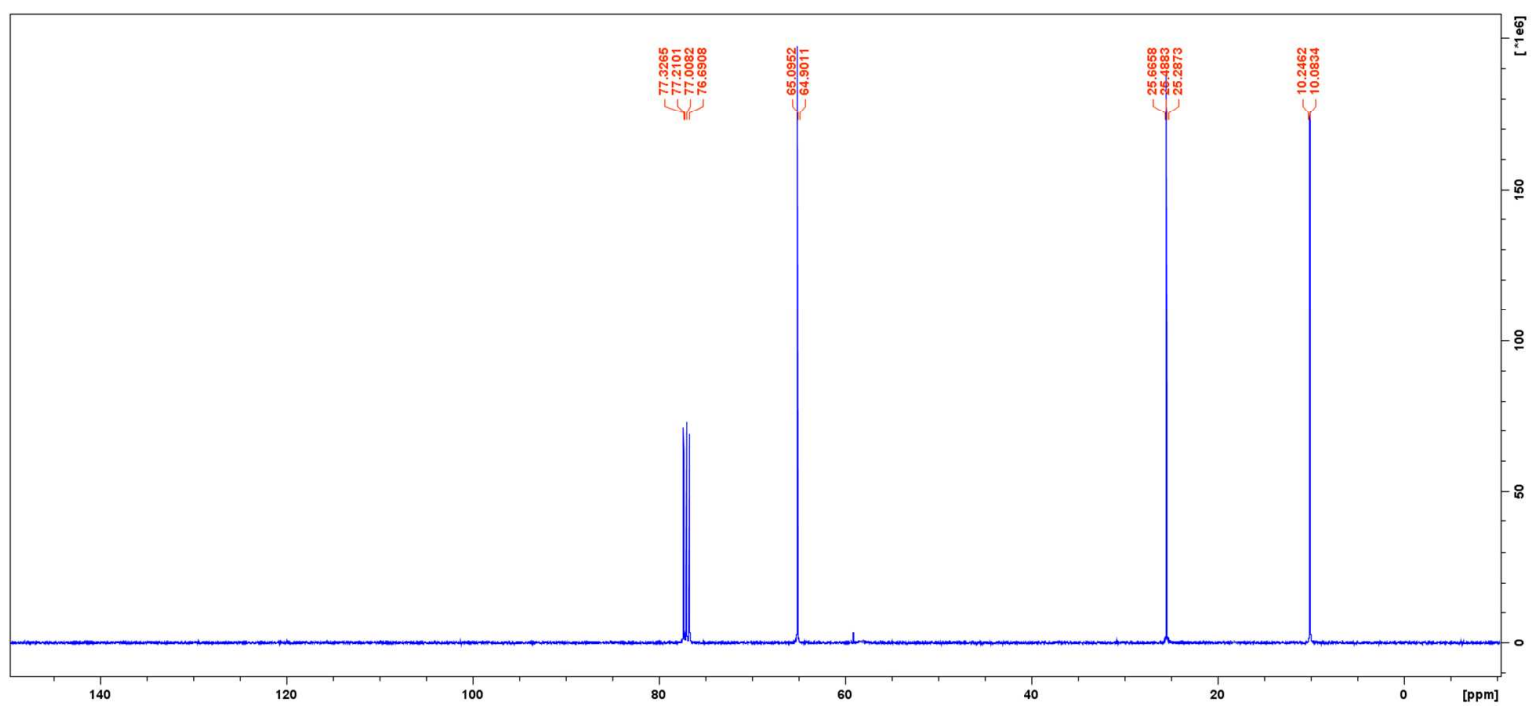

Figure S15. Tripropoxysilane ${ }^{13} \mathrm{C}$ NMR spectrum. 


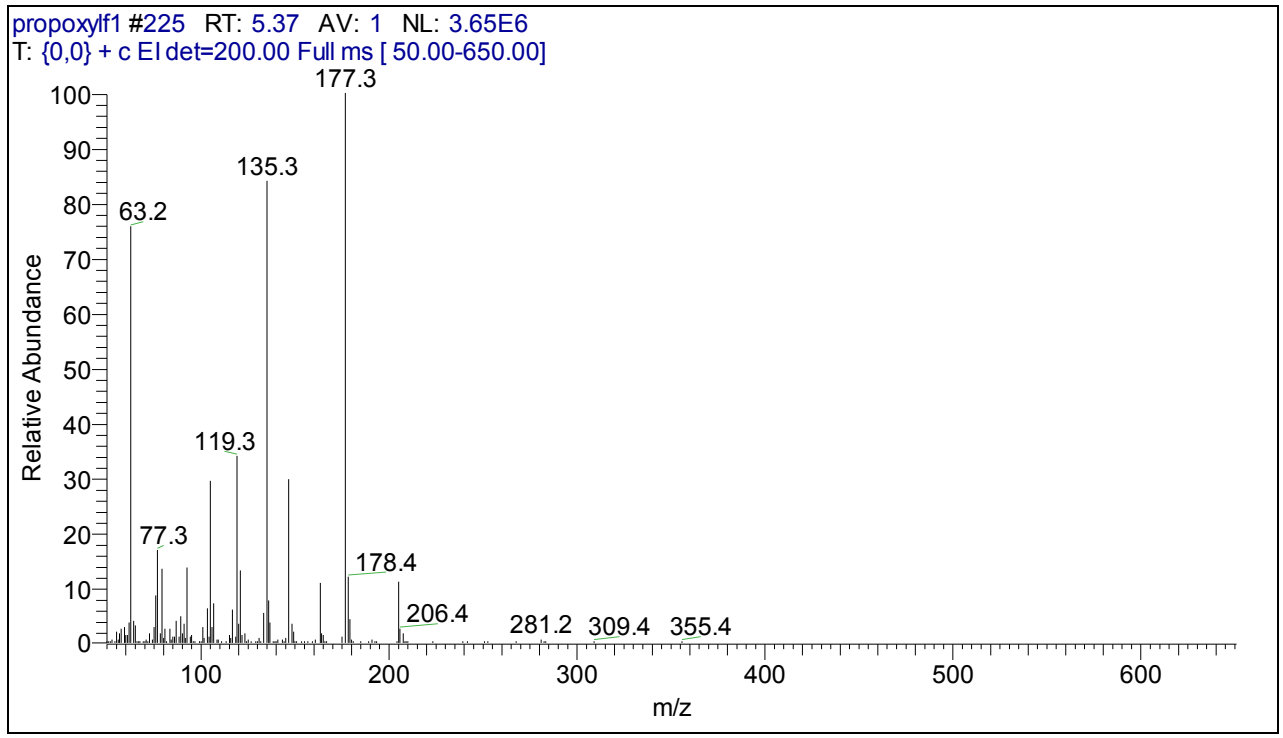

Figure S16. Tripropoxysilane GC-MS spectrum.

\section{Tetrapropoxysilane ${ }^{1} \mathrm{H}$ NMR, ${ }^{13} \mathrm{C}$ NMR and GC-MS}

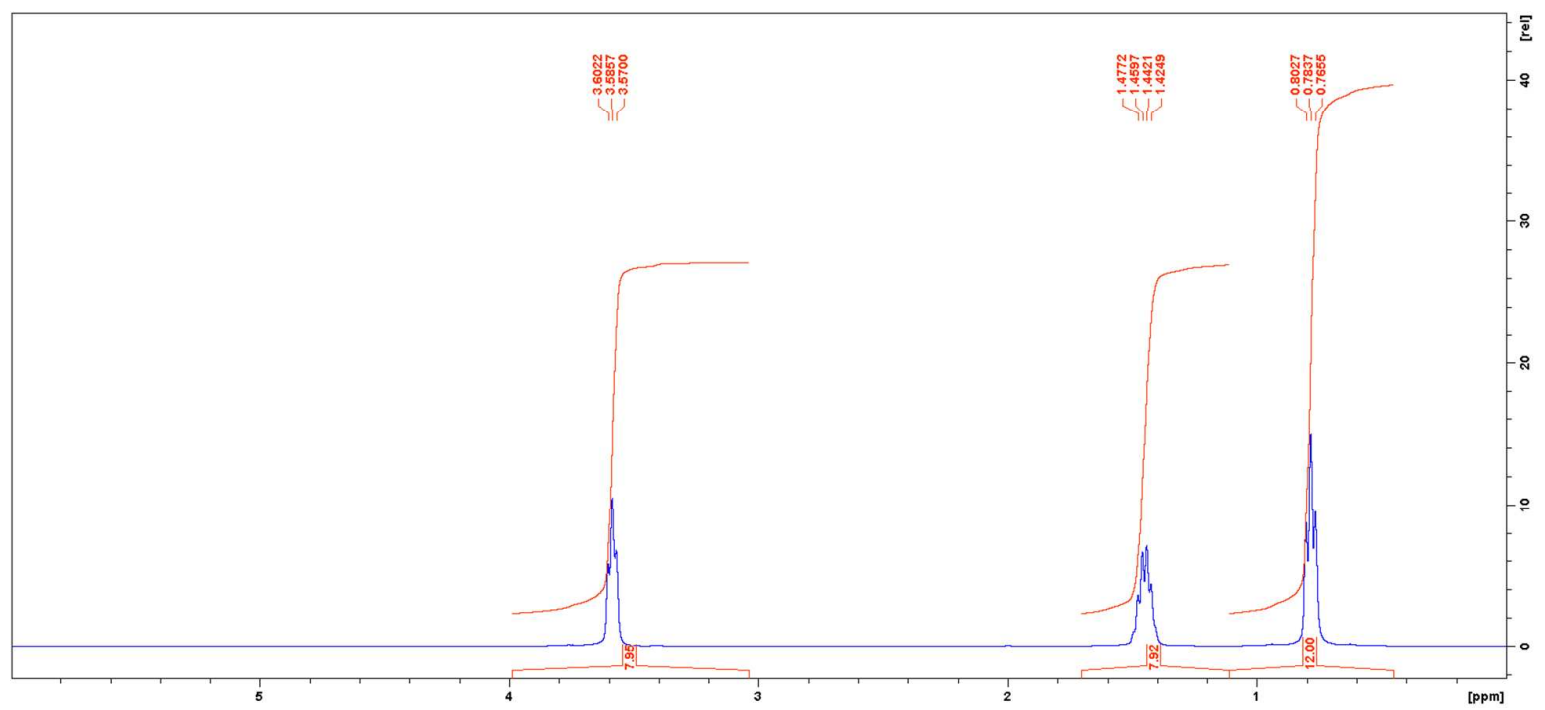

Figure S17. Tetrapropoxysilane ${ }^{1} \mathrm{H}$ NMR spectrum. 


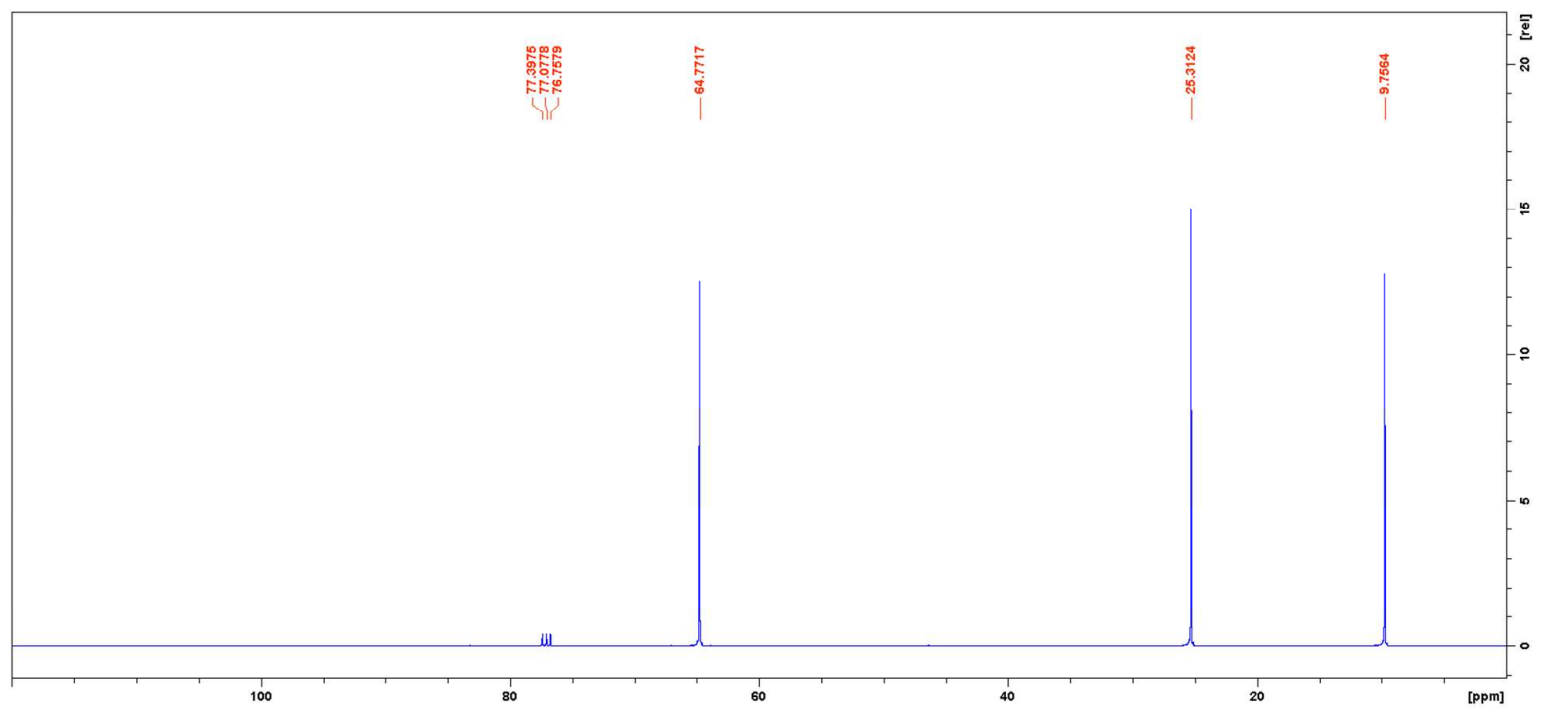

Figure S18. Tetrapropoxysilane ${ }^{13} \mathrm{C}$ NMR spectrum.

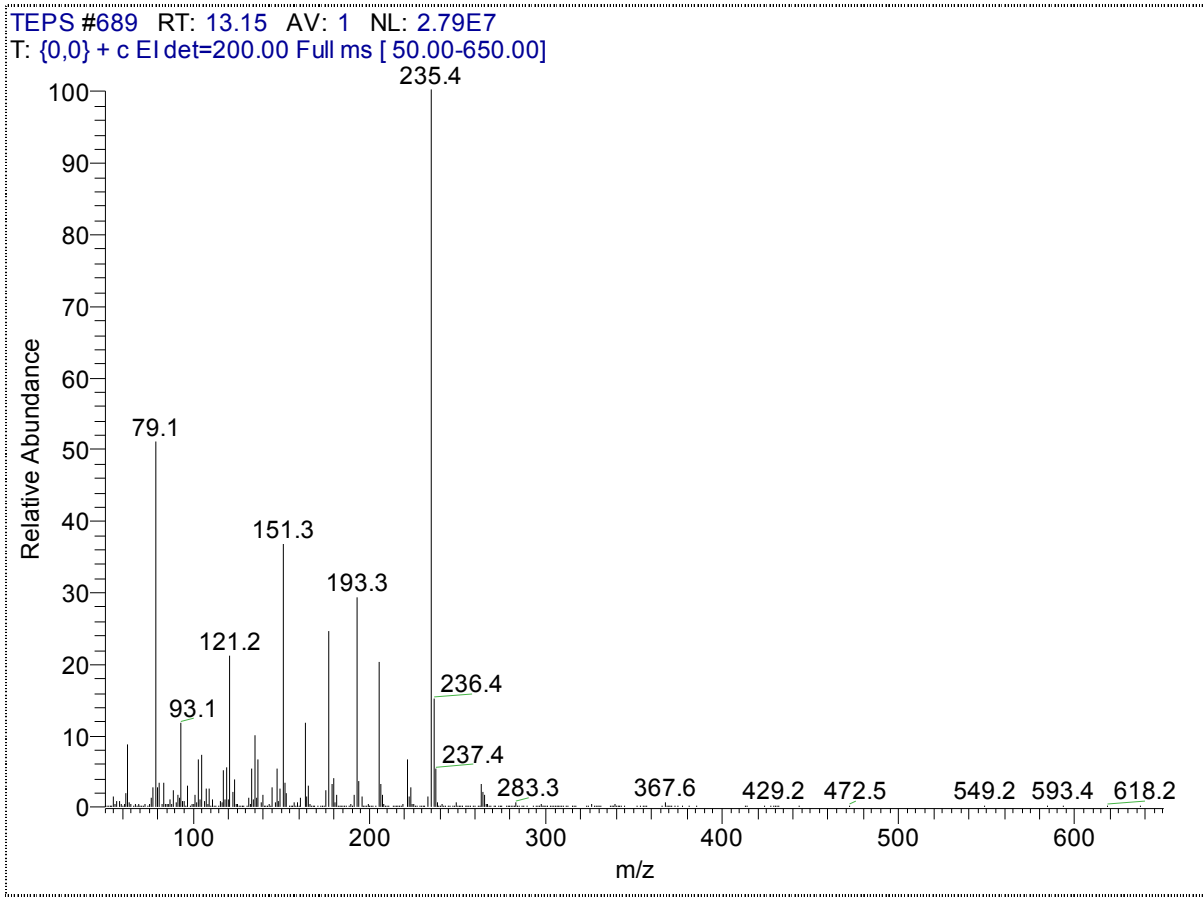

Figure S19. Tetrapropoxysilane GC-MS spectrum. 
Tributoxysilane ${ }^{1} \mathrm{H}$ NMR, ${ }^{13} \mathrm{C}$ NMR and GC-MS

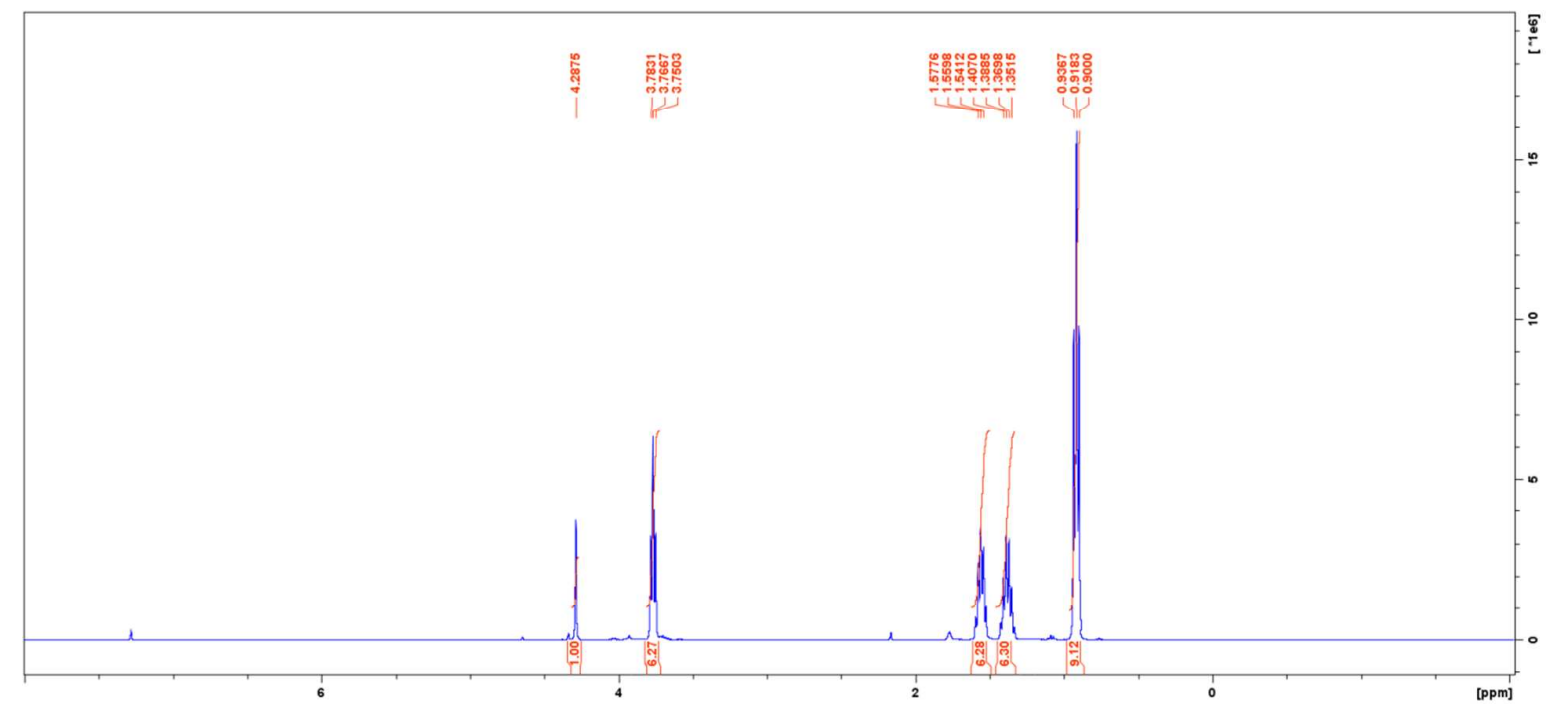

Figure S20. Tributoxysilane ${ }^{1} \mathrm{H}$ NMR spectrum.

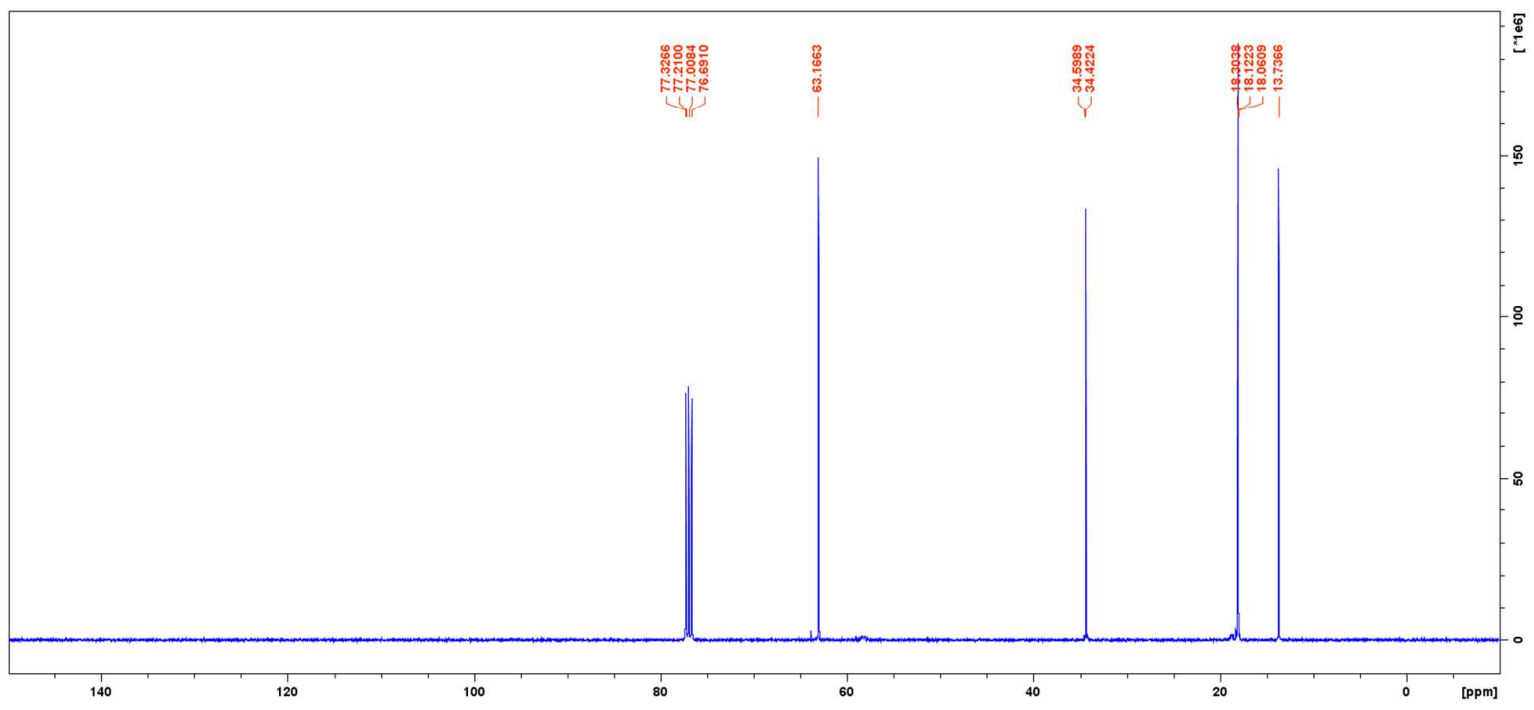

Figure S21. Tributoxysilane ${ }^{13} \mathrm{C}$ NMR spectrum. 


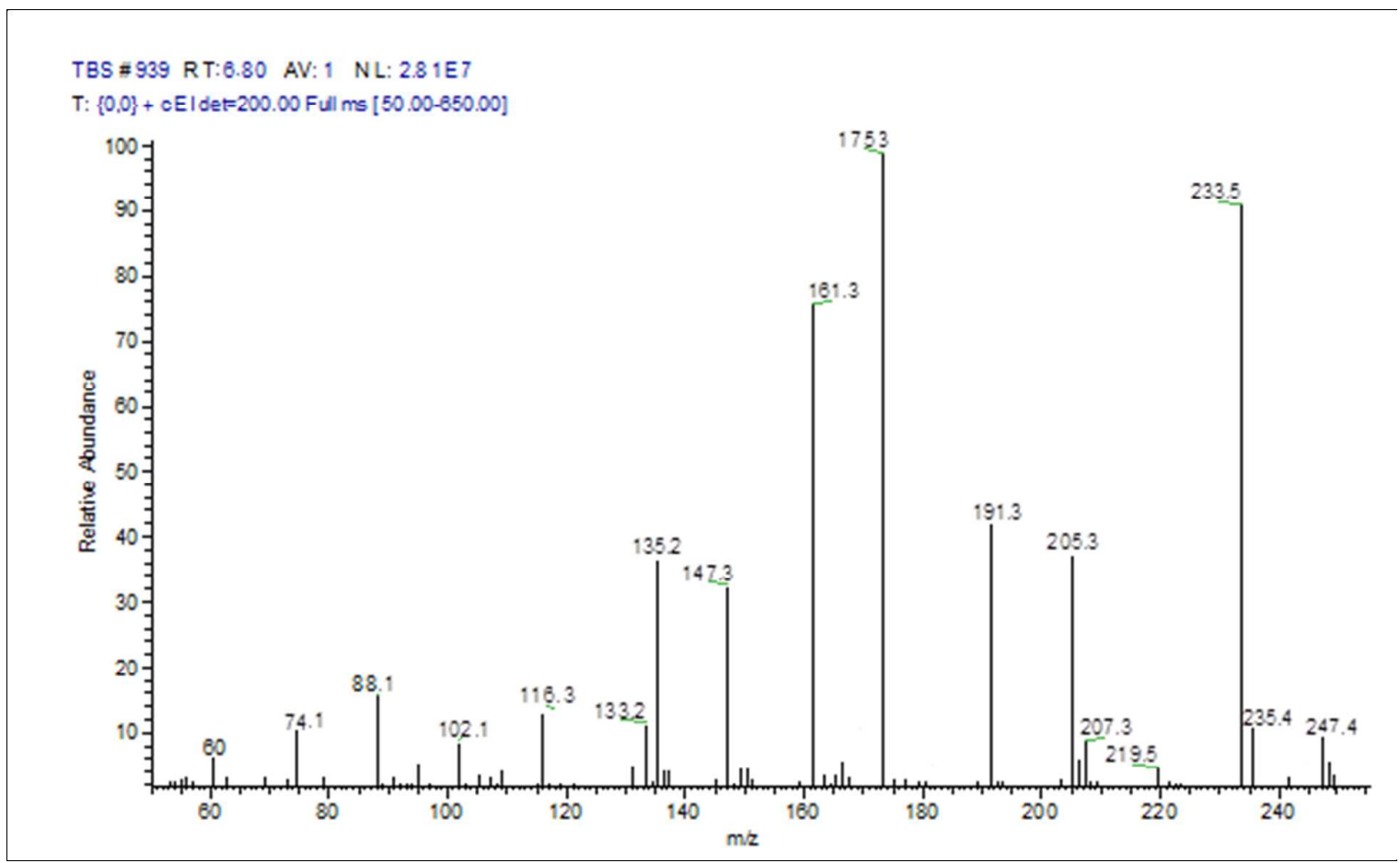

Figure S22. Tributoxysilane GC-MS spectrum.

Tetrabutoxysilane ${ }^{1} \mathrm{H}$ NMR, ${ }^{13} \mathrm{C}$ NMR and GC-MS

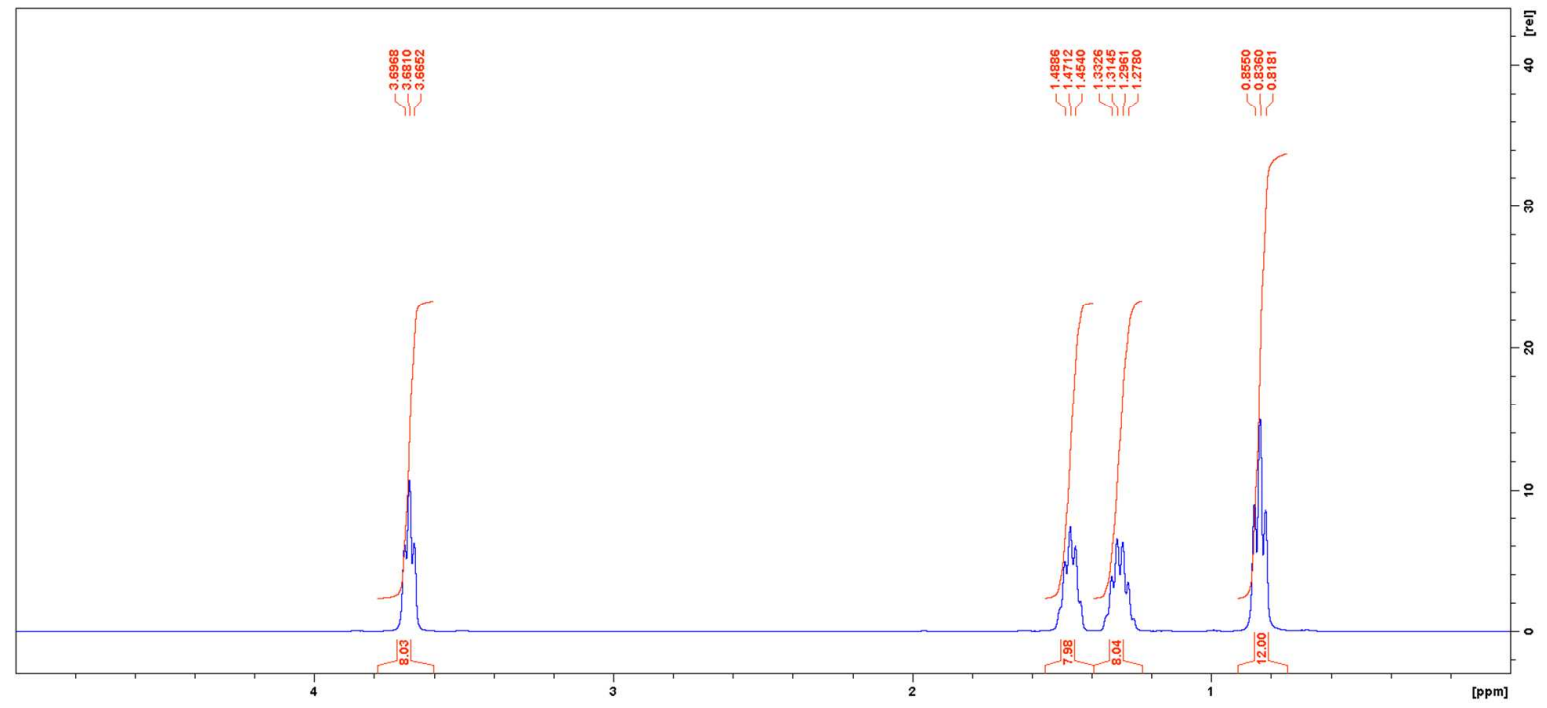

Figure S23. Tetrabutoxysilane ${ }^{1} \mathrm{H}$ NMR spectrum. 


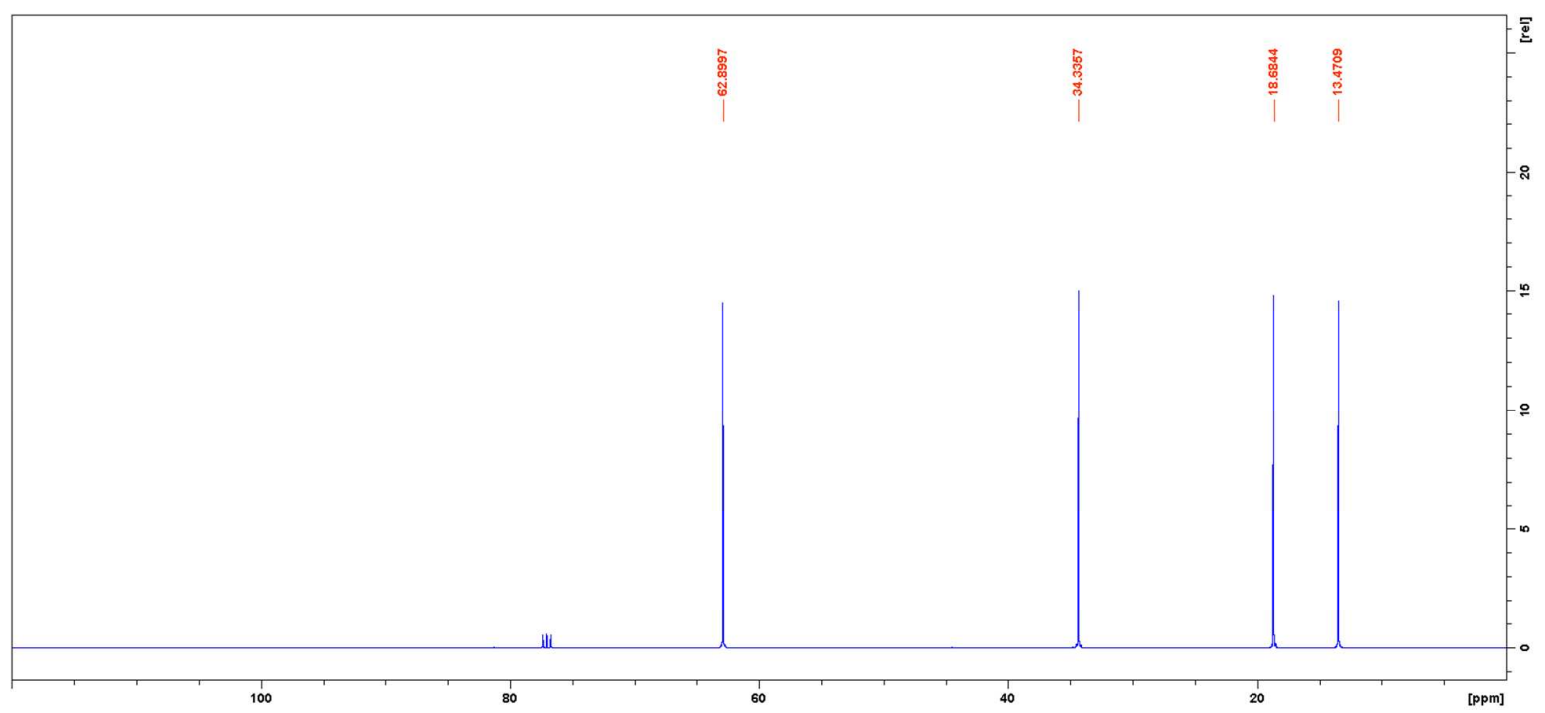

Figure S24. Tetrabutoxysilane ${ }^{13} \mathrm{C}$ NMR spectrum.

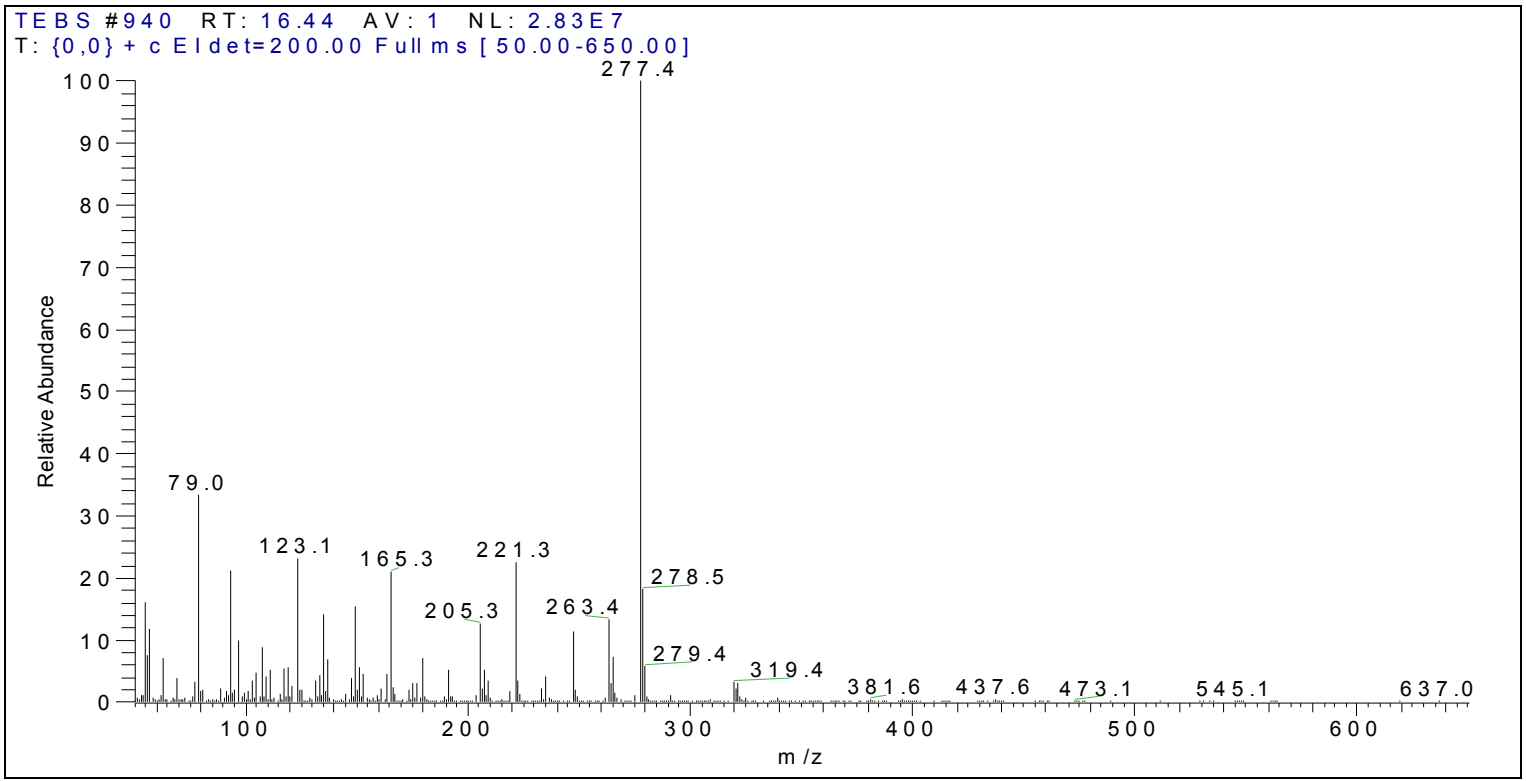

Figure S25. Tetrabutoxysilane GC-MS spectrum 
Table S1: Experimental design and results for synthesis in packed bed flow tubular reactor

\begin{tabular}{ccccc}
\hline $\begin{array}{c}\text { Experiment } \\
\text { number }\end{array}$ & $\begin{array}{c}\text { Ethanol flow } \\
\text { rate } \\
(\mathbf{m L} / \mathbf{m i n})\end{array}$ & $\begin{array}{c}\text { Reaction } \\
\text { temperature }\left({ }^{\circ} \mathrm{C}\right)\end{array}$ & $\begin{array}{c}\text { Observed Silicon } \\
\text { conversion (\%) }\end{array}$ & $\begin{array}{c}\text { Predicted Silicon } \\
\text { conversion (\%) }\end{array}$ \\
\hline 1 & 0.6 & 240 & 60.8 & 60.9 \\
2 & 0.9 & 230 & 57.3 & 57.2 \\
3 & 0.2 & 230 & 63.1 & 63.1 \\
4 & 0.6 & 180 & 54.9 & 54.8 \\
5 & 0.6 & 210 & 58.8 & 58.6 \\
6 & 1.0 & 210 & 54.0 & 54.1 \\
7 & 0.2 & 190 & 58.8 & 59.0 \\
8 & 0.1 & 210 & 62.6 & 62.4 \\
9 & 0.6 & 210 & 58.6 & 58.6 \\
10 & 0.6 & 210 & 58.7 & 58.6 \\
11 & 0.6 & 210 & 58.6 & 58.6 \\
12 & 0.9 & 190 & 53.1 & 53.1 \\
13 & 0.6 & 210 & 58.5 & 58.6 \\
\hline Reaction conditions: $5 \mathrm{wt} \% \mathrm{CuCl}$, activation time $2.5 \mathrm{~h}$, reaction period $24 \mathrm{~h}$ and activation temperature $220^{\circ} \mathrm{C}$.
\end{tabular}

Multiple regression output and predictive model equation

Table S2. Final multiple regression data for silicon conversion $\left(R^{2}=0.99\right)$

\begin{tabular}{cccccc}
\hline $\begin{array}{c}\text { Coefficient } \\
\text { symbol }\end{array}$ & Factor & $\begin{array}{c}\text { Estimated } \\
\text { coefficient }\end{array}$ & $\begin{array}{c}\text { Standard } \\
\text { error }\end{array}$ & $\boldsymbol{T}$-stat & $\boldsymbol{P}$-value \\
\hline$\beta_{0}$ & Intercept & 2.111 & 5.517 & 0.383 & 0.712 \\
$\beta_{1}$ & $\mathrm{X}_{1}$ (Flow rate) & -7.328 & 0.617 & -11.863 & $<0.0001$ \\
$\beta_{2}$ & $\mathrm{X}_{2}$ (Temperature) & 0.480 & 0.0524 & 9.146 & $<0.0001$ \\
$\beta_{3}$ & $\mathrm{X}_{1}{ }^{2}$ & -1.788 & 0.542 & -3.295 & $<0.0109$ \\
$\beta_{4}$ & $\mathrm{X}_{2}{ }^{2}$ & -0.0009 & 0.000125 & -7.209 & $<0.0001$ \\
\hline
\end{tabular}

The final predictive model equation fitted to silicon conversion was: 
$\%$ Silicon conversion $=2.111-7.328 \mathrm{X}_{1}+0.480 \mathrm{X}_{2}-1.788 \mathrm{X}_{1}^{2}-0.0009 \mathrm{X}_{2}^{2}$

Where, $\mathrm{X}_{1}=$ Flow rate and $\mathrm{X}_{2}=$ Reaction temperature.

Reaction kinetics for the alkoxylation reaction

Table S3. Silicon conversion data

\begin{tabular}{cccc}
\hline Time (h) & Si (\%) & $\mathbf{1 / [ \mathbf { A } ]}$ & $\ln [\mathbf{A}]$ \\
\hline 0 & 100 & 0.01 & 4.6052 \\
1 & 95 & 0.0105 & 4.5539 \\
2 & 92 & 0.0109 & 4.5218 \\
3 & 86 & 0.0116 & 4.4543 \\
4 & 81 & 0.0123 & 4.3944 \\
5 & 79 & 0.0127 & 4.3694 \\
6 & 75 & 0.0133 & 4.3175 \\
12 & 51 & 0.0196 & 3.9318 \\
24 & 36 & 0.0278 & 3.5835 \\
30 & 34 & 0.0294 & 3.5264 \\
\hline
\end{tabular}

Where; A is Silicon

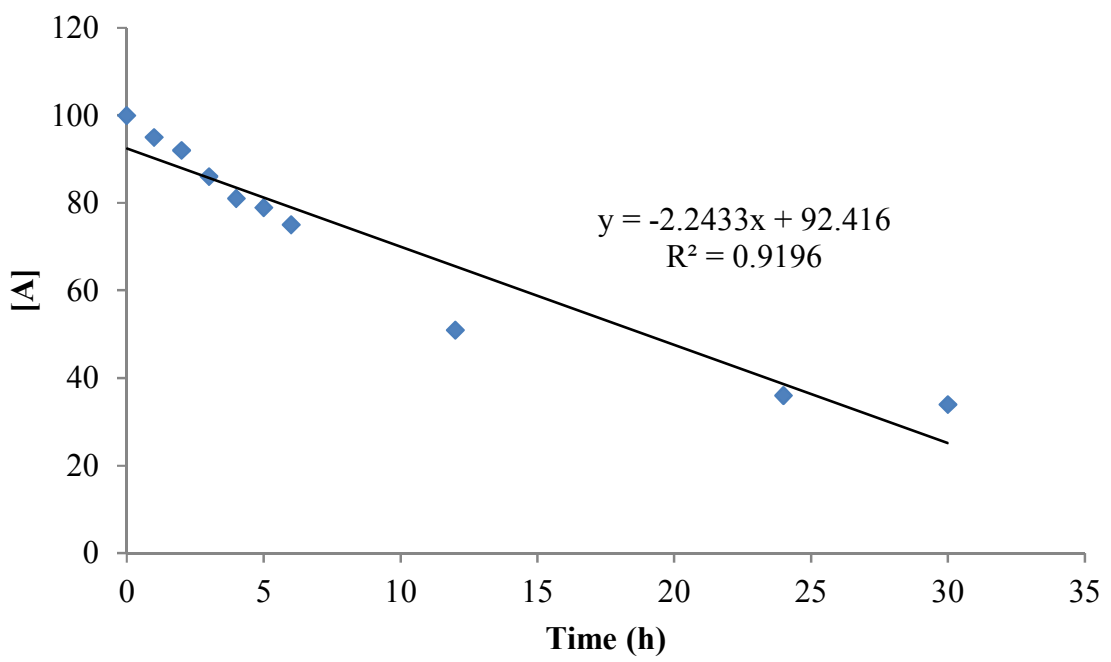

Figure S26. Zero order kinetic plot: where; A is Silicon 


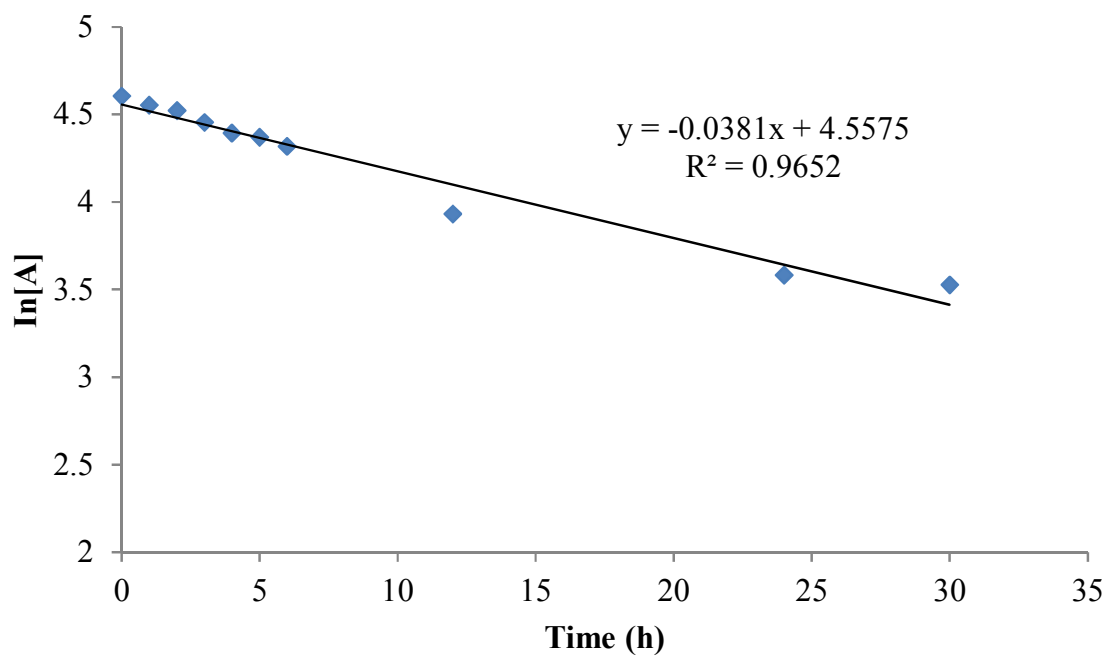

Figure S27. First order kinetic plot: where; A is Silicon

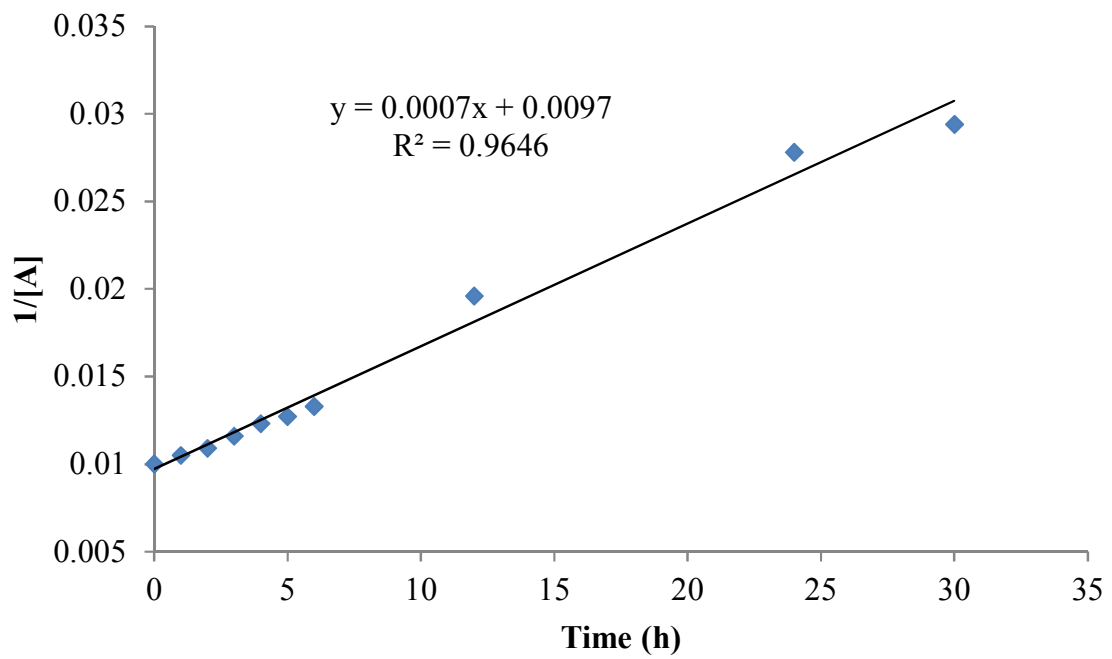

Figure S28. Second order kinetic plot: where; A is Silicon 


\section{Silicon conversion model validation graphs}

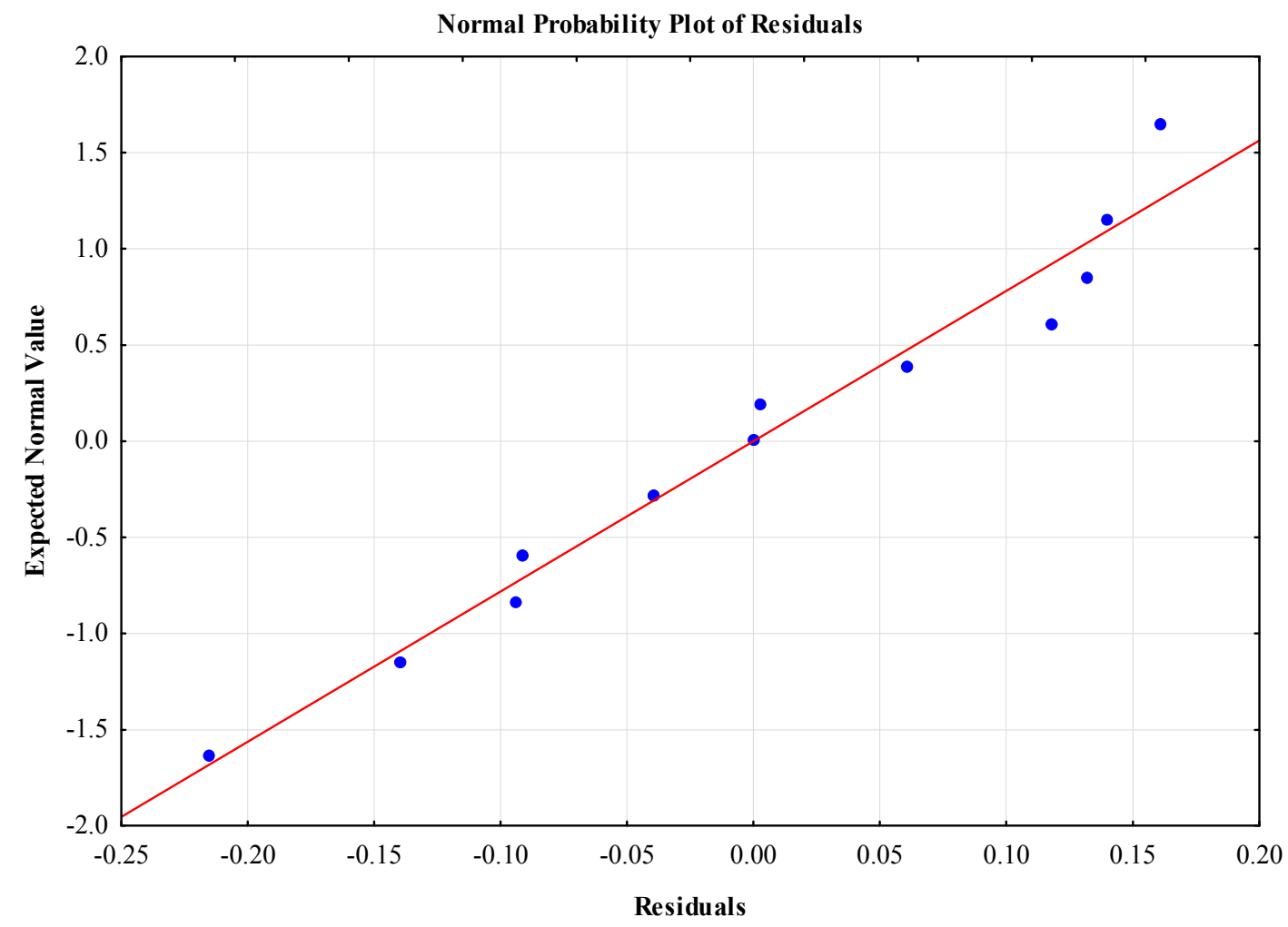

Figure S29. Normal probability plot for residuals for silicon conversion. 


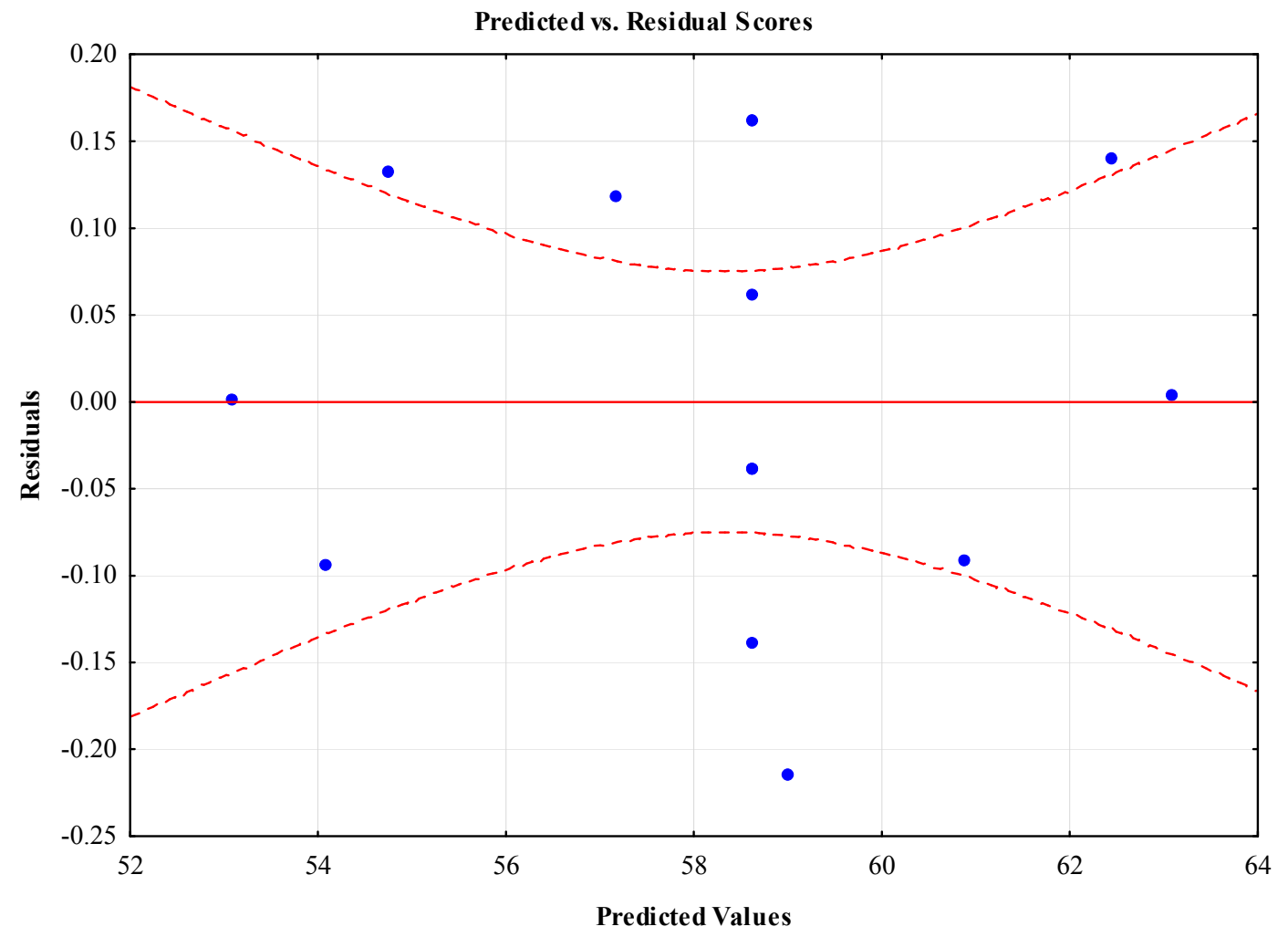

Figure S30. Outlier T plot of residuals for silicon conversion. 